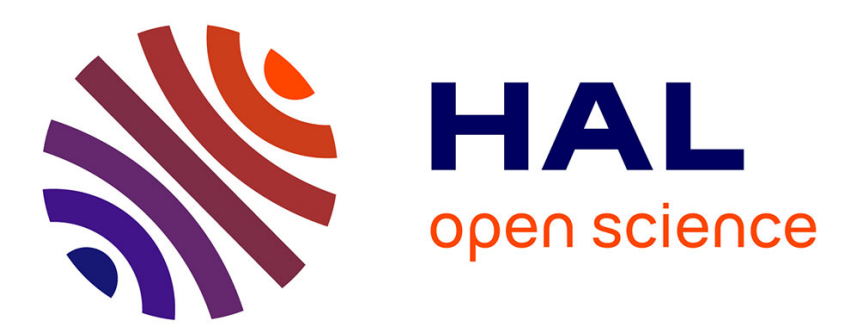

\title{
Iterative refinement of hierarchical T-meshes for bases of spline spaces with highest order smoothness
}

Dmitry Berdinsky, Tae-Wan Kim, Cesare Bracco, Durkbin Cho, Min-Jae Oh, Yeong-Hwa Seo, Sutipong Kiatpanichgij

\section{- To cite this version:}

Dmitry Berdinsky, Tae-Wan Kim, Cesare Bracco, Durkbin Cho, Min-Jae Oh, et al.. Iterative refinement of hierarchical T-meshes for bases of spline spaces with highest order smoothness. ComputerAided Design, 2014, 47, pp.96-107. 10.1016/j.cad.2013.10.004 . hal-03191235

\section{HAL Id: hal-03191235 \\ https://hal.science/hal-03191235}

Submitted on 7 Apr 2021

HAL is a multi-disciplinary open access archive for the deposit and dissemination of scientific research documents, whether they are published or not. The documents may come from teaching and research institutions in France or abroad, or from public or private research centers.
L'archive ouverte pluridisciplinaire HAL, est destinée au dépôt et à la diffusion de documents scientifiques de niveau recherche, publiés ou non, émanant des établissements d'enseignement et de recherche français ou étrangers, des laboratoires publics ou privés. 


\title{
Iterative refinement of hierarchical $\mathrm{T}$-meshes for bases of spline spaces with highest order smoothness
}

\author{
Dmitry Berdinsky ${ }^{\mathrm{a}, \mathrm{c}, \mathrm{d}}$, Tae-wan Kim ${ }^{\mathrm{a}, \mathrm{b}, *}$, Cesare Bracco ${ }^{\mathrm{e}}$, Durkbin Cho ${ }^{\mathrm{f}}$, \\ Min-jae $\mathrm{Oh}^{\mathrm{a}}$, Yeong-hwa Seo ${ }^{\mathrm{a}}$, Sutipong Kiatpanichgij ${ }^{\mathrm{b}}$, \\ ${ }^{a}$ Department of Naval Architecture and Ocean Engineering, Seoul National University, \\ Seoul 151-744, Republic of Korea \\ ${ }^{b}$ Research Institute of Marine Systems Engineering, Seoul National University, Seoul \\ 151-744, Republic of Korea \\ ${ }^{c}$ Department of Physics, Novosibirsk State University, Pirogova Str. 2, \\ 630090,Novosibirsk, Russia \\ ${ }^{d}$ Department of Computer Science, The University of Auckland, Auckland 1142, New \\ Zealand \\ ${ }^{e}$ Department of Mathematics "Giuseppe Peano", University of Turin, v. Carlo Alberto \\ 10, 10123, Turin, Italy \\ ${ }^{f}$ Department of Mathematics, Dongguk University, Pil-dong 3-ga, Jung-gu, Seoul \\ 100-715, Republic of Korea
}

\begin{abstract}
In this paper we propose a strategy for generating consistent hierarchical $\mathrm{T}$-meshes which allow local refinement and offer a way to obtain spline basis functions with highest order smoothness incrementally. We describe the required ordering of line-segments during refinement and the construction of spline basis functions. We give our strategy for generating consistent hierarchical T-meshes over any shape of a two-dimensional domain.
\end{abstract}

Keywords:

hierarchical T-mesh, spline space, local refinement

\footnotetext{
${ }^{*}$ Corresponding author

Email address: taewan@snu.ac.kr (Tae-wan Kim)

URL: http://caditlab.snu.ac.kr (Tae-wan Kim)
} 


\section{Introduction}

Local mesh refinement algorithms are useful for their ability to localize changes in the set of control points to a limited region; due to their construction, basic tensor-product representation does not allow local refinement. If we allow a row of control points to terminate at a $\mathrm{T}$-junction, we can refine an underlying domain locally. The spline representations based on a $\mathrm{T}$-mesh as an underlying structure appear to be efficient for surface modeling and isogeometric analysis introduced in 2005 by T.J.R. Hughes et al. [1, 2]. For the interested reader, let us give a short overview of some of the results related to the spline representation techniques using $\mathrm{T}$-meshes.

T-splines were introduced in 2003 by Sederberg et al. [3]. A practicable refinement algorithm of $\mathrm{T}$-splines is described in [4]. The issue of the linear independence of $\mathrm{T}$-splines [5], which is relevant to using $\mathrm{T}$-splines in isogeometric analysis [6], has been resolved lately by considering analysissuitable $\mathrm{T}$-splines by $\mathrm{Li}$ et al. [7]. The refinement algorithm for analysissuitable $\mathrm{T}$-splines is presented in [8]. Dual-compatible T-splines, which include analysis-suitable $\mathrm{T}$-splines, have been introduced in [9].

Multilevel B-splines for surface modeling were originally introduced in 1988 by Forsey and Bartels [10]. In 1995 Kraft [11] suggested a selection mechanism for hierarchical B-splines, which ensures their linear independence while permitting local control of refinement. Kraft's construction has been further elaborated in $[12,13]$. Dokken et al. [14] have lately proposed locally refinable splines (LR-splines) and the refining process based on so-called hand-in-hand LR-refinement which starts with a tensor-product mesh.

Polynomial splines over T-meshes were introduced in 2005 by Deng et al. [15]. PHT-splines [16], which are bicubic polynomial splines with the order of smoothness $(1,1)$ over T-meshes, were introduced in 2006. For applications of PHT-splines in surface modeling and isogeometric analysis see eg. $[17,18]$. Bases of spline spaces of bi-degree $\left(m, m^{\prime}\right)$ with the order of smoothness $\left(r, r^{\prime}\right)$ (if it is supposed that $m \geqslant 2 r+1$ and $m^{\prime} \geqslant 2 r^{\prime}+$ 1) over $\mathrm{T}$-meshes have been constructed in terms of the Bernstein-Bézeier representation by Schumaker et al. [19].

The issue of describing splines of bi-degree $\left(m, m^{\prime}\right)$ with the order of smoothness $\left(r, r^{\prime}\right)$ (if it is supposed that $m<2 r+1$ and $m^{\prime}<2 r^{\prime}+1$ ) over an arbitrary $\mathrm{T}-$ mesh seems hardly solvable in a simple and regular way. The dimension of the spline space may itself depend on the geometry of a T-mesh, and not only on its topology [20,21]. Therefore, it is desirable to gain an 
insight into the topology of $\mathrm{T}$-meshes which admit practicable algorithm for generating spline basis functions.

In this paper we will focus on a certain type of T-meshes with a multilevel structure. In particular, we will describe an iterative strategy to construct consistent hierarchical $\mathrm{T}$-meshes for splines with highest order smoothness; namely, for splines of bi-degree $(m, m)$ with the order of smoothness $(m-1, m-1)$. Wu et al. [22, 23] have recently introduced consistent hierarchical $\mathrm{T}$-meshes. A consistent hierarchical $\mathrm{T}$-mesh is generated by a sequence of edge insertions such that each new edge insertion into the mesh satisfies so-called surjection condition; in essence that means that one is able to add all new basis functions of a spline space with highest order smoothness at each iteration of edge insertion. The concept of a consistent hierarchical T-mesh seems to offer natural way to add spline basis functions incrementally. A consistent hierarchical $\mathrm{T}$-mesh might be generated for any shape of two-dimensional domain, and not just for rectangular domains. However, the generation of a consistent hierarchical $\mathrm{T}$-mesh and the ordering of the edges or line-segments remain open questions. As an implementation of a consistent hierarchical T-mesh, Wu et al. [22] suggest a particular type of crosswise hierarchical $\mathrm{T}$-mesh that contains a nested sequence of domains.

The essence of our iterative strategy can be described as follows. At each iteration step, the portion of a $\mathrm{T}$-mesh to be refined is chosen to be as follows: it creates a partition of some domain into cells of a tensor-product mesh. The partition of this domain into cells is of restricted configuration and the constraint on configuration will be given in terms of offset region and curve, which have been recently introduced by Giannelli and Jüttler [24]. We will determine the ordering of the line-segments to be inserted at each iteration step and give the construction of spline basis functions. This strategy provides framework in which the concept of consistent hierarchical $\mathrm{T}$-meshes is feasible. We will show that particular classes of consistent hierarchical Tmeshes [22] can be obtained by applying this strategy.

Overall, this paper is in the scope of the methods and tools introduced in [22], [24] and [25], and as a consequence the results presented can be considered as an extension of the construction of classical hierarchical Bsplines originally introduced in [11].

The rest of this paper is organized as follows. In Section 2 we provide the background needed to explain our strategy, which we then introduce at the beginning of Section 3. We describe the required ordering of line-segments and the construction of spline basis functions for splines of degrees 2 and 3 in 
Subsections 3.1 and 3.2 respectively. We make necessary remarks and discuss the relevant modifications of our strategy in Subsection 3.3. We conclude the paper in Section 4.

\section{Preliminaries}

A $T$-mesh $T$ is formed by a set of horizontal and vertical line-segments that creates a partition of a closed domain $\Omega$ into axis-aligned boxes, called the cells of the T-mesh, in which T-junctions are allowed [15]. We do not assume that the boundary $\partial \Omega$ of $\Omega$ is rectangular. However, we assume that a T-mesh $T$ is regular (see [19], Definition 2.2); or, in other words, we assume that the boundary $\partial \Omega$, which is a collection of piecewise-linear curves, is free of self-intersections.

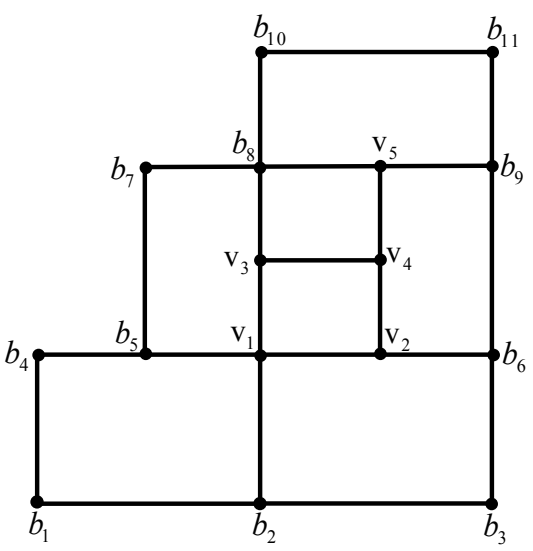

Figure 1: Example of a $\mathrm{T}-$ mesh.

An example of a T-mesh is shown in Fig.1. In Fig.1, $v_{1}, \ldots, v_{5}$ and $b_{1}, \ldots, b_{11}$ are the vertices of a $\mathrm{T}$-mesh. An edge of a $\mathrm{T}$-mesh is a linesegment connecting two adjacent vertices. In Fig. $1, b_{8} v_{5}$ and $v_{1} v_{2}$ are examples of horizontal edges and $b_{2} v_{1}$ and $v_{4} v_{5}$ are examples of vertical edges. The boundary of a T-mesh is the boundary $\partial \Omega$ of its domain $\Omega$. Thus in Fig.1 the boundary is the piecewise linear closed curve $b_{1} b_{3} b_{11} b_{10} b_{8} b_{7} b_{5} b_{4}$. A vertex $b$ lies on the boundary if $b \in \partial \Omega$; other vertices are called inner vertices.

A maximal segment of a $\mathrm{T}$-mesh is the longest possible line-segment that can be constructed from several collinear edges; in Fig.1 the line-segments $b_{4} b_{6}, b_{2} b_{10}$ are maximal. A maximal interior segment is a maximal segment 
that does not intersect with the boundary $\partial \Omega$; in Fig. $1 v_{3} v_{4}$ and $v_{2} v_{5}$ are maximal interior segments.

\subsection{Spline space with highest order smoothness over a T-mesh and smoothing cofactor method}

In Subsection 2.1 we will state the results that will be used in analysis of spline space with highest order smoothness defined as follows.

Definition 1 ([15]). Let $S(m, m, m-1, m-1)(T)$ be a space of functions $f(x, y)$ of class $C^{m-1, m-1}(\Omega)$ which are polynomials of bi-degree $(m, m)$ in $x$ and $y$, defined in each cell of a $T$-mesh $T$.

To analyze the dimension and basis functions of a space $S(m, m, m-$ $1, m-1)(T)$, we follow the smoothing cofactor method [20, 26, 27].

For a given element $f(x, y) \in S(m, m, m-1, m-1)(T)$ and inner vertex $v\left(x_{0}, y_{0}\right)$, the conformality factor $k_{v}$ is defined as follows. Suppose that the bivariate function $f(x, y)$ is respectively equal to the polynomials $P_{1}(x, y)$, $P_{2}(x, y), P_{3}(x, y), P_{4}(x, y)$ in each of four cells which form a neighborhood of $v\left(x_{0}, y_{0}\right)$ (see Fig.2). In the case of $\mathrm{T}$-junction the corresponding polynomials are supposed to be the same.

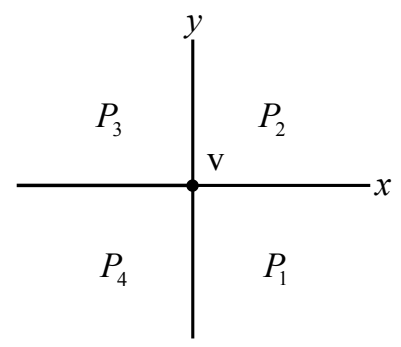

Figure 2: Four cells adjacent to the vertex $v$.

Provided that the function $f(x, y)$ is of class $C^{m-1}$ with respect to $x$ and $y$, the following equalities hold:

$$
\begin{aligned}
& P_{2}-P_{1}=\lambda_{1,2}(x)\left(y-y_{0}\right)^{m}, \\
& P_{3}-P_{2}=\lambda_{2,3}(y)\left(x-x_{0}\right)^{m}, \\
& P_{4}-P_{3}=\lambda_{3,4}(x)\left(y-y_{0}\right)^{m}, \\
& P_{1}-P_{4}=\lambda_{4,1}(y)\left(x-x_{0}\right)^{m},
\end{aligned}
$$


for some univariate polynomials $\lambda_{1,2}(x), \lambda_{2,3}(y), \lambda_{3,4}(x)$ and $\lambda_{4,1}(y)$ of degree $m$. From equations (1) to (4), we can assert:

$$
\begin{aligned}
P_{2}-P_{1}+P_{4}-P_{3}= & \left(\lambda_{1,2}(x)+\lambda_{3,4}(x)\right)\left(y-y_{0}\right)^{m}= \\
& -\left(\lambda_{2,3}(y)+\lambda_{4,1}(y)\right)\left(x-x_{0}\right)^{m} .
\end{aligned}
$$

Thus, for some constant $k_{v}$ we obtain:

$$
\lambda_{1,2}(x)+\lambda_{3,4}(x)=k_{v}\left(x-x_{0}\right)^{m}, \lambda_{2,3}(y)+\lambda_{4,1}(y)=-k_{v}\left(y-y_{0}\right)^{m} .
$$

In addition, the constant $k_{v}$ can be represented in terms of the derivatives on the left-hand side of (5). Indeed, from (5) and (6) we can obtain the following representation for $k_{v}$ :

$$
k_{v}=\left.\frac{1}{(m !)^{2}} \frac{\partial^{2 m}\left(P_{2}(x, y)+P_{4}(x, y)-P_{1}(x, y)-P_{3}(x, y)\right)}{\partial x^{m} \partial y^{m}}\right|_{x=x_{0}, y=y_{0}} .
$$

This means that $k_{v}$ is uniquely defined for a given $f \in S(m, m, m-1, m-$ 1)(T) and inner vertex $v$.

Suppose that $E$ is a maximal horizontal interior segment with endpoints at the vertices $v_{1}, v_{n}$, and at the inner vertices $v_{2}, \ldots, v_{n-1}$ (see Fig.3). For a given function $f \in S(m, m, m-1, m-1)(T)$, let $P_{i j}(x, y), i=1 \ldots n$, $j=1 \ldots 4$ denote the polynomials that correspond to the vertices $v_{i}$, following Fig.2.

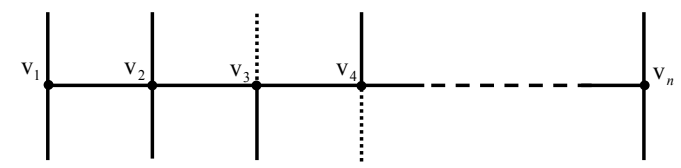

Figure 3: The maximal interior segment $E$. The vertical dashed lines mean that these are T-junctions at the points $v_{3}$ and $v_{4}$.

We can then note the following relation:

$$
\sum_{i=1}^{n} P_{i 2}-P_{i 1}+P_{i 4}-P_{i 3}=0
$$

From equations (5),(6) and (8), we obtain the zero identity:

$$
\sum_{i=1}^{n} k_{v_{i}}\left(x-x_{i}\right)^{m} \equiv 0
$$


where $x_{i}, i=1 \ldots n$ is the $x$-coordinate of the vertex $v_{i}$. This identity (9) is equivalent to the following linear system in terms of the conformality factors $k_{v_{i}}, i=1 \ldots n$ :

$$
\left\{\begin{array}{l}
\sum_{i=1}^{n} k_{i}=0, \\
\sum_{i=1}^{n} k_{i} x_{i}=0, \\
\cdots \\
\sum_{i=1}^{n} k_{i} x_{i}^{m}=0 .
\end{array}\right.
$$

An analogous linear system for a maximal vertical interior segment can be derived:

$$
\left\{\begin{array}{l}
\sum_{i=1}^{n} k_{i}=0, \\
\sum_{i=1}^{n} k_{i} y_{i}=0, \\
\ldots \\
\sum_{i=1}^{n} k_{i} y_{i}^{m}=0 .
\end{array}\right.
$$

Definition 2 ([22]). For a given T-mesh $T$, let $v_{1}, \ldots, v_{N}$ be vertices of $T$ belonging to maximal interior segments of $T$. The conformality vector space $W[T]$ is the space of conformality factors $k_{v_{1}}, \ldots, k_{v_{N}}$ which satisfy the linear systems (10) and (11) for all horizontal and vertical maximal interior segments.

Note that, unlike the similar definition given elsewhere [22, 23], this does not involve the conformality factors of boundary vertices.

Thus, by virtue of (10) and (11), and using the direct formula (7), it is possible to define a linear map correctly $[22,23]$ :

$$
K: S(m, m, m-1, m-1)(T) \longrightarrow W[T] .
$$

The definition of a conformal vector space in the univariate case is the following:

Definition 3 ([22]). Given a univariate knot vector $\left(x_{1}, \ldots, x_{n}\right)$ without repeated knots, and the corresponding line-segment $E=\left[x_{1}, x_{n}\right], W[E]$ is the space of conformality factors $k_{1}, \ldots, k_{n}$ which satisfy the linear system (10).

Note that the space $W[E]$ is isomorphic to the space of functions of the class $C^{m-1}$ which are polynomials of degree $m$ within each segment $\left[x_{j}, x_{j+1}\right]$, $j=1 \ldots n-1$ and identically zero outside the segment $E=\left[x_{1}, x_{n}\right]$; following 
previous authors $[22,23]$, we call this space $\bar{S}(m, m-1)(E)$. If we further suppose that $f(x) \in \bar{S}(m, m-1)(E)$ such that $f(x)=P_{j}(x)$, when $x \in$ $\left[x_{j}, x_{j+1}\right], j=1 \ldots n-1$ and $f \equiv 0$, for $x \in \mathbb{R} \backslash\left[x_{1}, x_{n}\right]$. Since $f(x)$ is of class $C^{m-1}$, we can write

$$
\begin{array}{r}
P_{1}-0=k_{1}\left(x-x_{1}\right)^{m}, P_{2}-P_{1}=k_{2}\left(x-x_{2}\right)^{m}, \ldots, \\
P_{n-1}-P_{n-2}=k_{n-1}\left(x-x_{n-1}\right)^{m}, 0-P_{n-1}=k_{n}\left(x-x_{n}\right)^{m} .
\end{array}
$$

This implies that $\sum_{i=1}^{n} k_{i}\left(x-x_{i}\right)^{m} \equiv 0$; and the conformality factors $k_{1}, \ldots, k_{n}$ can be obtained by solving the linear system (10). Note that $\operatorname{dim} W[E]=$ $n-m-1$ if $n>m+1$, and $\operatorname{dim} W[E]=0$ if $n \leqslant m+1$.

Definition 4 ([22]). Let $E$ be a maximal interior segment in the T-mesh $T$. Then we define $P_{E}: W[T] \rightarrow W[E]$ to be the projection that preserves the conformality factors of the vertices belonging to $E$.

Indeed, by Definition 2, the conformality factors of the vertices of a linesegment $E$ satisfy the system (10) for any conformality vector in $W[T]$. We are ready to prove the following simple lemma:

Lemma 1. For a maximal interior segment $E$ of a given $T$-mesh $T$, let $T^{\prime}$ denote the new $T$-mesh that is obtained from $T$ by removing line-segment $E$. Assume that there is a set of functions $f_{1}, \ldots, f_{l} \in S(m, m, m-1, m-1)(T)$ such that the conformality vectors $P_{E}\left(K\left(f_{1}\right)\right), \ldots, P_{E}\left(K\left(f_{l}\right)\right)$ form the basis of a subspace $P_{E}(\operatorname{im} K) \subset W[E]$, where $\operatorname{im} K \subset W[T]$. Then the spline space $S(m, m, m-1, m-1)(T)$ can be expressed as follows:

$S(m, m, m-1, m-1)(T)=S(m, m, m-1, m-1)\left(T^{\prime}\right) \bigoplus \operatorname{span}\left\{f_{1}, \ldots, f_{l}\right\}$

Proof: The equality $S(m, m, m-1, m-1)\left(T^{\prime}\right) \cap \operatorname{span}\left\{f_{1}, \ldots, f_{l}\right\}=\mathbf{0}$ follows from the simple observation that $P_{E}\left(K\left(f^{\prime}\right)\right)=0$ for any $f^{\prime} \in S(m, m, m-$ $1, m-1)\left(T^{\prime}\right)$. It is then only necessary to represent the spline function $f \in S(m, m, m-1, m-1)(T)$ as the sum of a spline function $g \in S(m, m, m-$ $1, m-1)\left(T^{\prime}\right)$ and some linear combination of $f_{1}, \ldots, f_{l}$.

For a given function $f \in S(m, m, m-1, m-1)(T)$, the corresponding projection $P_{E}(K(f))$ is a linear combination of $P_{E}\left(K\left(f_{i}\right)\right), i=1 \ldots l$; thus, for some $c_{1}, \ldots, c_{l}$, the projection $P_{E}\left(K\left(f-\sum_{i=1}^{l} c_{i} f_{i}\right)\right)=0$. Thus the function $g=f-\sum_{i=1}^{l} c_{i} f_{i}$ is an element of $S(m, m, m-1, m-1)\left(T^{\prime}\right)$. Indeed, since $P_{E}(K(g))=0$, all the conformality factors of $K(g)$ belonging to $E$ 
are zero. Without loss of generality, we may suppose that $E$ is the horizontal maximal interior segment that traverses the vertices $v_{1}, \ldots, v_{n}$ (see Fig.3), and that all its conformality factors $k_{v_{1}}, \ldots, k_{v_{n}}$ are zero. Following Fig. $2, P_{i j}, i=1 \ldots n, j=1 \ldots 4$ are polynomials representing $f$ in the neighborhoods of the vertices $v_{1}, \ldots, v_{n}$ respectively. Since the $\mathrm{T}-$ mesh $T$ has a T-junction at the vertex $v_{1}, P_{13}-P_{14}=0$ and, by virtue of (5) and (6), $P_{12}-P_{11}=0$, or equivalently $P_{23}-P_{24}=0$; since $k_{v_{2}}=0$, a similar argument yields $P_{22}-P_{21}=0$. Going further, to the vertex $v_{n}$, we see that $g$ is of class $C^{\infty}$ with respect to $y$ along the whole line-segment $E$. Thus $g \in S(m, m, m-1, m-1)\left(T^{\prime}\right)$ and $f=g+\sum_{i=1}^{l} c_{i} f_{i}$. The Lemma 1 is proved.

Remark 1. Note that, if the projection $P_{E}: \operatorname{im} K \rightarrow W[E]$ is surjective, then $\operatorname{span}\left\{f_{1}, \ldots, f_{l}\right\}$ is isomorphic to $W[E]$.

Corollary 1. Suppose that for a given T-mesh $T$, there is an ordered sequence of maximal interior segments $E_{1} \prec \cdots \prec E_{n}$, such that successive deletion of $E_{n} \succ \cdots \succ E_{1}$ from the $T$-mesh $T$ produces the $T$-mesh $T_{0}$; let the $T$-mesh $T_{i}, i=1 \ldots n-1$ be obtained by successive deletion of the line-segments $E_{n} \succ \cdots \succ E_{i+1}$ from $T$; and let $T_{n}=T$. Suppose that all the projections $P_{E_{i}}: \operatorname{im} K_{i} \rightarrow W\left[E_{i}\right], i=1 \ldots n$ are surjective, where linear maps $K_{i}: S(m, m, m-1, m-1)\left(T_{i}\right) \rightarrow W\left[T_{i}\right]$ are given by (12). Let $\left\{f_{i 1}, \ldots, f_{i l_{i}}\right\} \subset S(m, m, m-1, m-1)\left(T_{i}\right), i=1 \ldots n$ be a set of spline functions such that the projections $P_{E_{i}}\left(K_{i}\left(f_{i j}\right)\right), j=1 \ldots l_{i}$ form the basis of $W\left[E_{i}\right]$. Then, by virtue of Lemma 1 :

$$
\begin{array}{r}
S(m, m, m-1, m-1)(T)=S(m, m, m-1, m-1)\left(T_{0}\right) \\
\bigoplus_{i=1}^{n} \operatorname{span}\left\{f_{i 1}, \ldots, f_{i l_{i}}\right\} .
\end{array}
$$

One consequence of Corollary 1 is a method of constructing basis functions of a spline space for those classes of $\mathrm{T}$-meshes that satisfy the conditions of the corollary.

This approach to the construction of basis functions, provided by corollary 1 but originally introduced by $\mathrm{Wu}$ et al. [22], has been formulated [23] there in terms of ordered sequence of edges, rather than maximal interior segments. But in demonstrating their concept of consistent hierarchical T-meshes, Wu et al. [23] refer only to a particular class of hierarchical T-meshes [22]. In the 
next section we will discuss the problem of generating $\mathrm{T}$-meshes to satisfy the conditions of Corollary 1, and then to construct basis functions of the corresponding spline space.

Definition 5 ([25]). For a given ordered sequence of maximal interior segments $E_{1} \prec \cdots \prec E_{l}$, let $\lambda\left(E_{i}\right)$ be the number of vertices of $E_{i}$ which are not on a maximal interior segment of index greater than $i$.

We will use Lemma 2 below in the proof of Lemma 4 in Subsection 3.2.

Lemma 2 ([25]). Let $T$ be a T-mesh on some rectangular domain. Assume that, for a given order of its maximal interior segments, the following inequality holds:

$$
\lambda\left(E_{i}\right) \geqslant m+1,
$$

for every maximal interior segment $E_{i}, i=1 \ldots l$. Then,

$$
\operatorname{dim} S(m, m, m-1, m-1)(T)=(m+1)^{2} C-m(m+1) E^{0}+m^{2} V^{0},
$$

where $C$ is the number of cells, $E^{0}$ is the number of inner edges, and $V^{0}$ is the number of inner vertices of $T$.

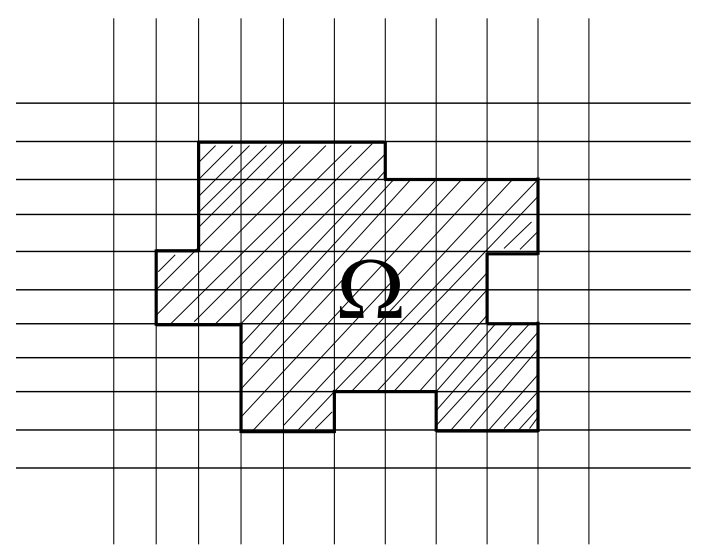

Figure 4: A hatched domain $\Omega$ formed by the cells of a tensor-product mesh. 


\subsection{Offset from a domain}

In Subsection 2.2 we will recall Giannelli and Jüttler's definition [24] of the offset region and curve from a domain $\Omega$ that will be necessary to describe the refinement strategy in Section 3. In addition, we will state the results that will be used in Lemmas 3 and 4 in Subsections 3.1 and 3.2, respectively.

Suppose that $\Omega$ is a domain formed by the cells of an infinite tensorproduct mesh (see Fig.4). The offset region $R_{k}$ can be defined recursively as follows: an offset region $R_{k+1}$ is defined to be the set of cells $\nsubseteq \bigcup_{j=0}^{k} R_{j}$ which have at least one point in common with the boundary $\partial \bigcup_{j=0}^{k} R_{j}$; the zero offset region $R_{0}$ is defined to be $\Omega$. The offset curve from a domain $\Omega$ is the piecewise linear curve defined as follows:

Definition 6 ([24]). The offset curve $C_{0}$ at a distance 0 from a domain $\Omega$ is defined to be its boundary $\partial \Omega$. Given the offset regions $R_{k}$ and $R_{k+1}$, for a nonnegative integer $k$, then:

1. We say that $\Omega$ admits an offset at a distance $k+\frac{1}{2}$ if any cell in $R_{k+1}$ is related to $R_{k}$ by one of the three connections shown in Fig.5; then the offset curve at a distance $k+\frac{1}{2}$, written $C_{k+\frac{1}{2}}$, is a piecewise linear curve obtained by collecting together the contributions of every cell, as shown in Fig.5.

2. We say that $\Omega$ admits an offset at a distance $k+1$ if the relationship between any cell in $R_{k+1}$ and $R_{k}$ falls into one of the three cases shown in Fig.6; then the offset curve at a distance $k+1$, written $C_{k+1}$, is the piecewise linear curve that forms the exterior boundary of $R_{k+1}$, as shown in Fig.6.

We say that $\Omega$ does not admit an offset at a distance greater than or equal to $k+\frac{1}{2}$ or $k+1$ if the above conditions 1 or 2 respectively are not satisfied.

In Definition 6 the conditions 1 or 2 respectively allow us to be sure that the offset curves $C_{k+\frac{1}{2}}$ or $C_{k+1}$ are free of self-intersections. Fig.7 shows an example of the offset region $R_{1}$ from the domain $\Omega$ (shown in Fig.4 as well) and the corresponding offset curve $C_{\frac{1}{2}}$ (dashed red lines). Note that the definition above means that $\Omega$ in Fig. 7 does not admit an offset at a distance greater or equal than 1 .

Definition 6 constraints the configuration of a domain $\Omega$ in a way which makes it relatively easy to specify the basis functions of a spline space over a T-mesh. 

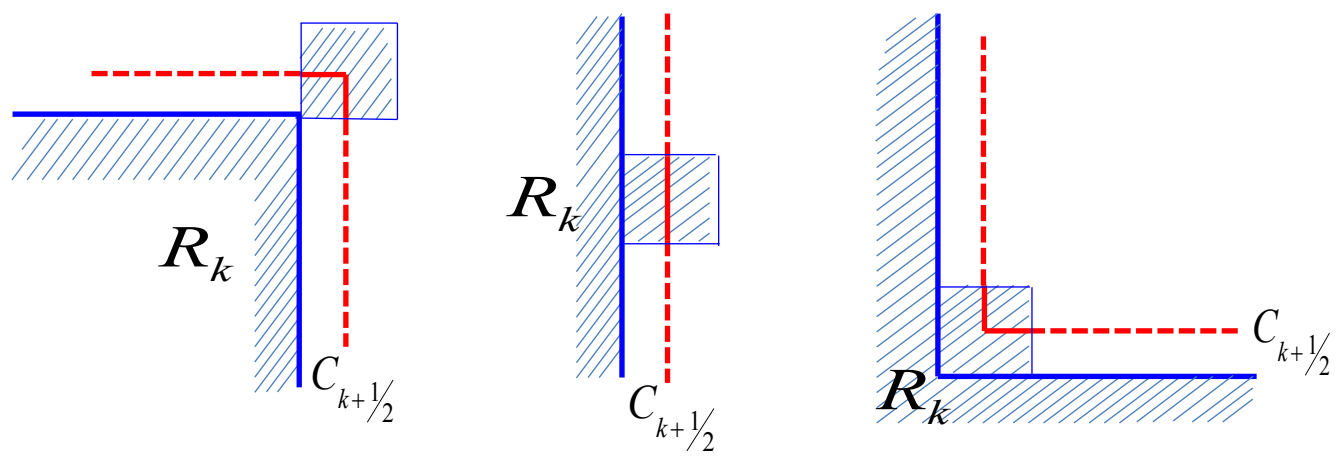

Figure 5: Admissible connections between a cell of the offset region $R_{k+1}$ and $R_{k}$ : a shared corner (left), one shared side (center) and two shared sides (right). The piecewise linear contributions to the offset curve resulting from each of the three cases are the solid portions of the red lines. (For interpretation of the references to color in this figure legend, the reader is referred to the web version of this article.)
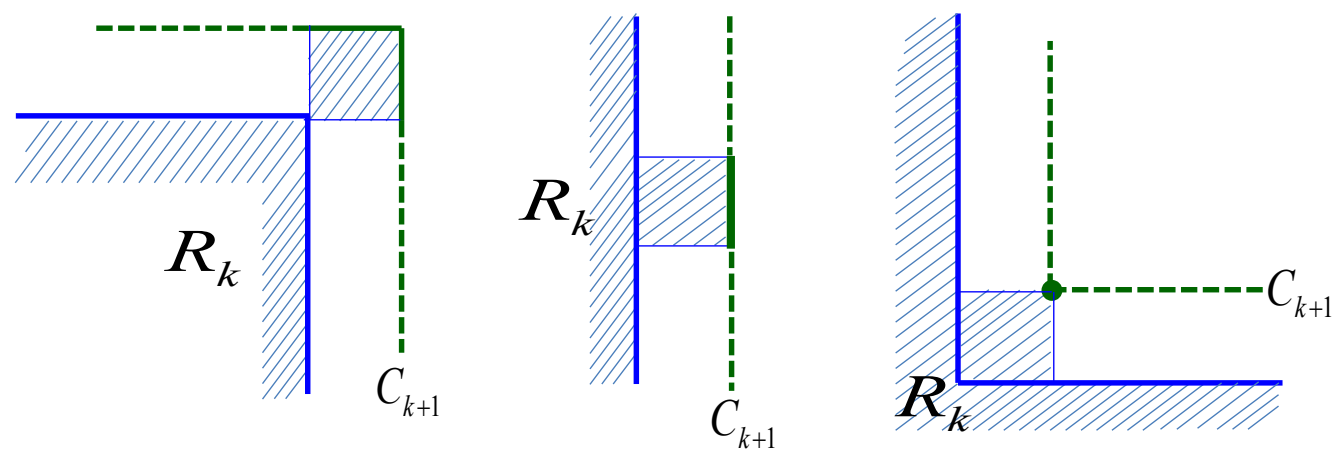

Figure 6: Admissible connections between a cell of the offset region $R_{k+1}$ and $R_{k}$ : a shared corner (left), one shared side (center) and two shared sides (right). Two adjacent sides (left), one side (center) and one dot (right), which are shown as the solid portions of the green lines, must be free, where free means that the edge or the vertex belongs to the boundary of $\bigcup_{j=0}^{k+1} R_{j}$. The piecewise linear contributions to the offset curve resulting from each of the three admissible cases are also shown as the solid portions of the green lines, and the green dot. (For interpretation of the references to color in this figure legend, the reader is referred to the web version of this article.) 


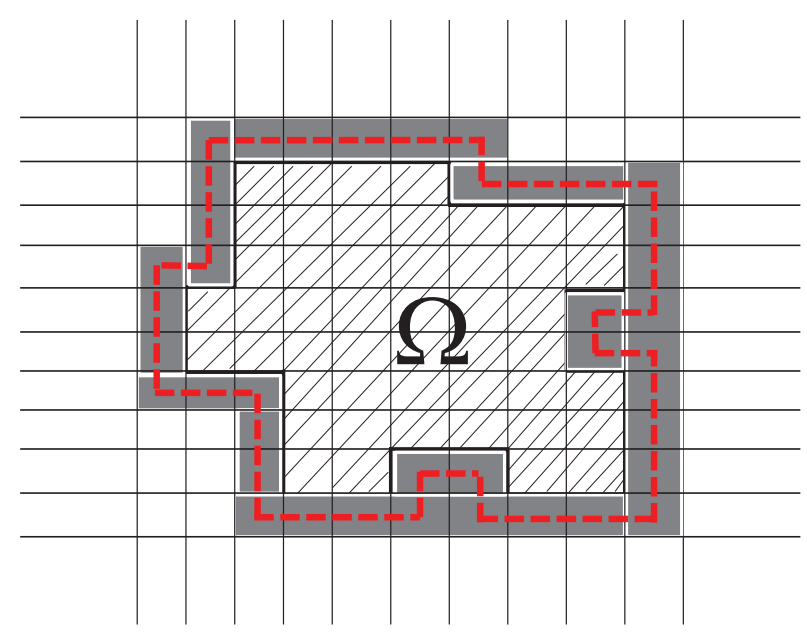

Figure 7: The region $R_{1}$ (gray cells) is the offset region from the domain $\Omega$; the corresponding offset curve $C_{\frac{1}{2}}$ is shown as red dashed lines. (For interpretation of the references to color in this figure legend, the reader is referred to the web version of this article.)

Theorem 1 ([24]). Let $T$ be a T-mesh which subdivides a bounded domain $\Omega$ into cells of a tensor-product mesh (see Fig.4). For a given integer $m \geqslant 2$, suppose that $\Omega$ admits an offset at a distance $\frac{m-1}{2}$. Let $\mathcal{B}$ be a set of tensorproduct B-splines generated from the given tensor-product mesh. Then, the following set provides the basis of the spline space $S(m, m, m-1, m-1)(T)$ :

$$
\left\{\left.\tau\right|_{\Omega}: \tau \in \mathcal{B} \wedge \operatorname{supp} \tau \cap \operatorname{int} \Omega \neq \varnothing\right\}
$$

The following definition of the configuration of a domain $\Omega$, which is the dual of Definition 6, will be used extensively in the next section.

Definition 7. Let $m$ be an integer $\geqslant 2$. We say that a domain $\Omega$ admits an inner offset at a distance $\frac{m-1}{2}$ if its complement $\mathbb{R}^{2} \backslash \Omega$ admits an offset at a distance $\frac{m-1}{2}$. We say that a domain $\Omega$ does not admit an inner offset at a distance greater than or equal to $\frac{m-1}{2}$ if its complement $\mathbb{R}^{2} \backslash \Omega$ does not admit an offset at a distance greater than or equal to $\frac{m-1}{2}$. The inner offset curve of $\Omega$ at a distance $\frac{m-1}{2}$ is $C_{\frac{m-1}{2}}$ of $\mathbb{R}^{2} \backslash \Omega$.

A condition that $\Omega$ admits an inner offset at a distance $\frac{m-1}{2}$ allows us to be sure that the inner offset curve of $\Omega$ at a distance $\frac{m-1}{2}$ is free of selfintersections. Examples of domains which admit inner offset at distances $\frac{1}{2}$ 

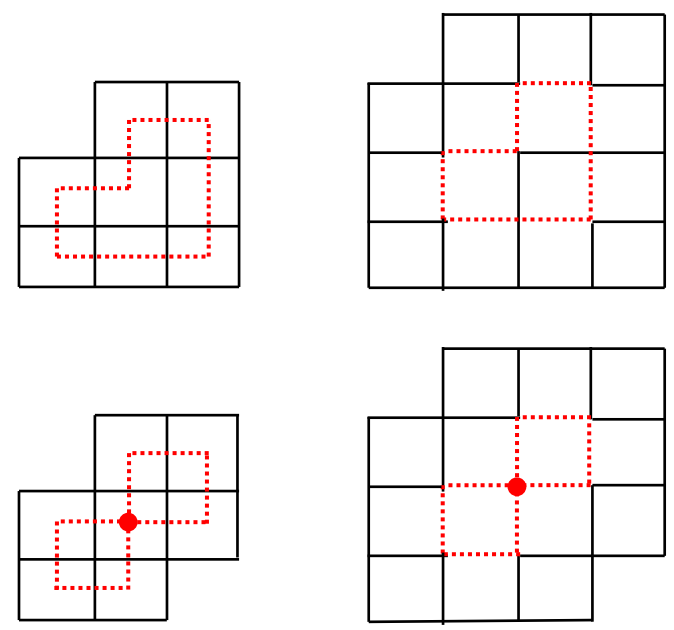

Figure 8: Domains which admit inner offset at distances $\frac{1}{2}$ and 1 are shown at the top left and right respectively; domains which do not admit inner offset at distances grater than or equal to $\frac{1}{2}$ and 1 are shown at the bottom left and right respectively. The inner offset curves are the red dashed lines and self-intersections are red dots. (For interpretation of the references to color in this figure legend, the reader is referred to the web version of this article.

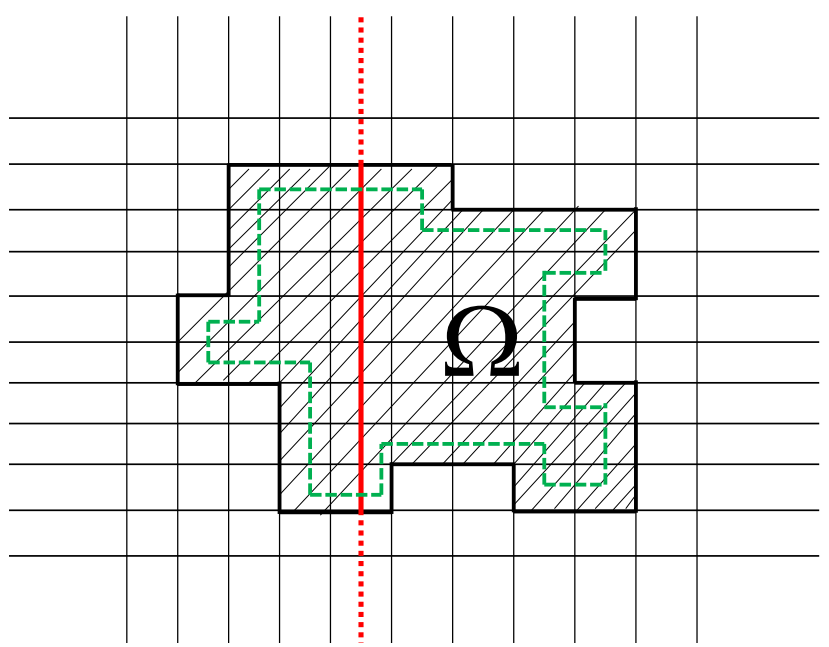

Figure 9: A line-segment $\Omega \cap L$ is shown as a red, solid line; its extension up to line $L$ is shown as red, dashed lines. The inner offset curve at a distance $\frac{1}{2}$ from the domain $\Omega$ for the refined tensor-product mesh is shown with green dashed lines. (For interpretation of the references to color in this figure legend, the reader is referred to the web version of this article.) 
and 1 are shown in Fig.8, together with domains which do not admit an inner offset at distances greater than or equal to $\frac{1}{2}$ and 1 .

We can make the following simple observation about a domain which admits an inner offset:

Proposition 1. For a given integer $m \geqslant 2$, suppose that a domain $\Omega$ admits an inner offset at a distance $\frac{m-1}{2}$. Further suppose that the corresponding tensor-product mesh is refined by inserting a line L. Then, $\Omega$ admits an inner offset at a distance $\frac{m-1}{2}$ for the refined tensor-product mesh as well.

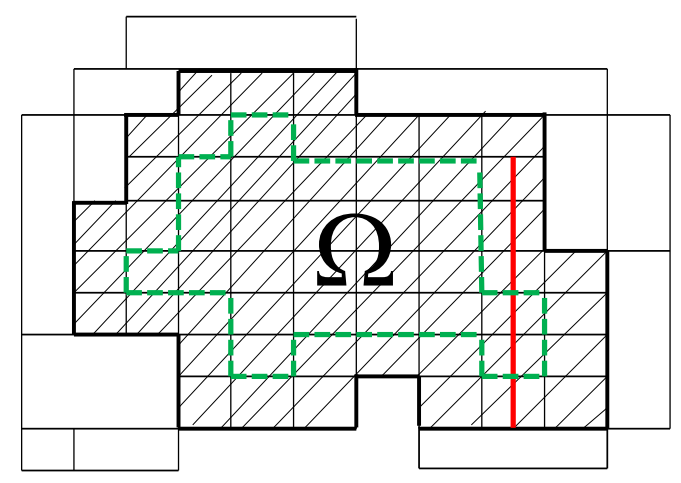

Figure 10: The line-segment $E$ is shown as a red line; the inner offset curve at a distance 1 from the domain $\Omega$ is shown as green dashed lines. (For interpretation of the references to color in this figure legend, the reader is referred to the web version of this article.)

Proposition 1 is illustrated, for $m=2$, in Fig.9. The following proposition plays a significant role in proving lemmas in the next section.

Proposition 2. Let $T^{\prime}$ be a T-mesh and $\Omega$ be a domain composed by a subset of cells of $T^{\prime}$. Suppose that the set of cells comprising $\Omega$ is a subset of cells of some tensor-product mesh. For a given integer $m \geqslant 2$, we suppose that $\Omega$ admits an inner offset at a distance $\frac{m-1}{2}$. Suppose further that $T^{\prime}$ is refined by the line-segment $E \subset \Omega$ such that $E$ is a maximal interior segment of the refined $T$-mesh $T=T^{\prime} \cup E$. Then we can assert:

$S(m, m, m-1, m-1)(T)=S(m, m, m-1, m-1)\left(T^{\prime}\right) \bigoplus \operatorname{span}\left\{f_{1}, \ldots, f_{l}\right\}$,

where $f_{1}, \ldots, f_{l}$ are tensor-product B-splines which have supports within the domain $\Omega$. 
Proof: Before proving Proposition 2 let us illustrate its nature, for the case $m=3$, in Fig.10. We will prove the proposition for $m=3$; the proof for other positive integers $m$ is analogous. Following Lemma 1, we need to provide tensor-product B-splines $f_{1}, \ldots, f_{l} \in S(m, m, m-1, m-1)(T)$ such that $P_{E}\left(K\left(f_{1}\right)\right), \ldots, P_{E}\left(K\left(f_{l}\right)\right)$ form a basis of $P_{E}(\mathrm{im} K) \subset W[E]$. Without loss of generality, we assume that $E$ is the vertical maximal interior segment, and let $y_{1}<\cdots<y_{k}$ be the $y$-coordinates of its knots. If $k \leqslant 4$, then $W[E]=\mathbf{0}$ and there is nothing to prove. If $k>4$, then there are $l=k-4$ tensorproduct B-splines $f_{1}, \ldots, f_{l}$ from the spline space $S(m, m, m-1, m-1)(T)$, such that $P_{E}\left(K\left(f_{1}\right)\right), \ldots, P_{E}\left(K\left(f_{l}\right)\right)$ form a basis of $W[E]$. Let us consider
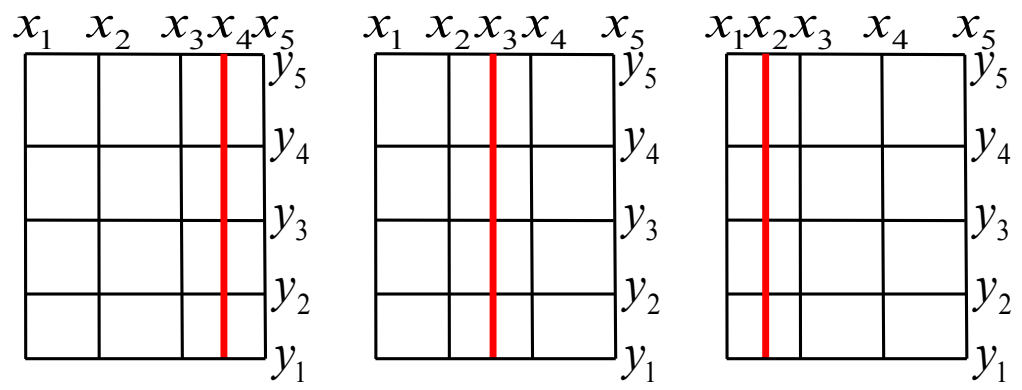

Figure 11: The cells of the domain $\Omega$ are shown in black, and line-segment $V$ is the red line. (For interpretation of the references to color in this figure legend, the reader is referred to the web version of this article.)

a column of four adjacent cells divided by $E$ and the corresponding linesegment $V$ with knots $\left(y_{1}, y_{2}, y_{3}, y_{4}, y_{5}\right)$.

Since $\Omega$ admits an inner offset at a distance 1 , then at least one of the three configurations shown in Fig.11 must occur. Consider the following tensor-product B-spline having a support within the domain $\Omega$ (see Fig.11):

$$
f(x, y)=N\left[x_{1}, x_{2}, x_{3}, x_{4}, x_{5}\right](x) N\left[y_{1}, y_{2}, y_{3}, y_{4}, y_{5}\right](y) ;
$$

then $P_{E}(K(f(x, y)))$ is a nontrivial element $\left(k_{1}, \ldots, k_{5}\right)$ of the one-dimensional space of conformality factors $W[V] \subset W[E]$. Indeed, $k_{i} \neq 0, i=1 \ldots 5$, because otherwise $\left(k_{1}, \ldots, k_{5}\right)=0$ and then, as we saw in the proof of Lemma $1, f(x, y)$ would be of class $C^{\infty}$ with respect to $x$ along the line-segment $E$, which contradicts the definition of $f(x, y)$ in (16). From this simple treatment, it is readily apparent that the conformality vectors $P_{E}\left(K\left(f_{i}\right)\right), i=$ $1 \ldots l$ for the B-splines $f_{i}, i=1 \ldots l$ corresponding to all rows of four con- 
secutive cells divided by $E$, form a basis of the space $W[E]$. Proposition 2 is proved.

\section{Refinement strategy}

We now describe general refinement strategy that satisfies the conditions introduced in Corollary 1, which allow spline basis functions to be constructed incrementally. We will concentrate on $m=2$ and $m=3$. However, we will start by describing the generic inductive refinement procedure.

Definition 8. Let $m$ be an integer $\geqslant 2$. We say that a T-mesh $T$ is of type $m$ if it is derived from an initial $T-m e s h T_{0}$ by repeating step 2 of the following algorithm from $j=0$ until $j=N-1$ and $T=T_{N}$, for some integer $N \geqslant 1$.

1. The initial T-mesh $T_{0}$ occupies the domain $\Omega_{0}$, which is composed of the cells of a tensor-product mesh and admits an offset at a distance $\frac{m-1}{2}$.

2. Induction from any $j \geqslant 0$ to $j+1$ proceeds as follows. For a given T-mesh $T_{j}$, it is necessary to find domains $\widehat{\Omega}_{j} \subset \Omega_{j}$ which satisfy the following assumptions: domains $\widehat{\Omega}_{j}$ and $\Omega_{j}$ are both constructed from the cells of some tensor-product mesh, such that each cell in $\Omega_{j}$ is also a cell of the T-mesh $T_{j}$; and $\Omega_{j}$ admits an inner offset at a distance $\frac{m-1}{2}$. The refined $T$-mesh $T_{j+1}$ is derived from $T_{j}$ by dividing each cell of the domain $\widehat{\Omega}_{j}$ following the rule illustrated in Fig.12, such that the line-segments $E_{j 1}, \ldots, E_{j n_{j}}$ refining $T_{j}$ have endpoints on $\partial \widehat{\Omega}_{j}$. It is supposed that the line-segments $E_{j 1}, \ldots, E_{j n_{j}}$ are maximal interior segments of the T-mesh $T_{j+1}$, i.e. $E_{j 1}, \ldots, E_{j n_{j}}$ do not intersect with the boundary $\partial \Omega_{0}$.

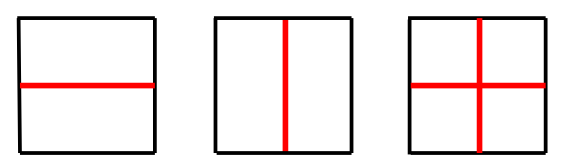

Figure 12: Three types of subdivision of a cell: horizontal (left), vertical (center), and crosswise (right). (For interpretation of the references to color in this figure legend, the reader is referred to the web version of this article.) 
A step 2 for an iteration from $j$ to $j+1$ for $m=3$ is shown in Fig.13. The examples of realization of the refinement strategy are demonstrated in Remarks 2 and 3 below for the cases $m=2$ and $m=3$, respectively.

Since $\Omega_{0}$ admits an offset at a distance $\frac{m-1}{2}$, the basis functions of a spline space $S(m, m, m-1, m-1)\left(T_{0}\right)$ can always be obtained using Theorem 1. In order to show that a $\mathrm{T}$-mesh $T$ of type $m$ satisfies the the conditions of Corollary 1 , we only need to prove that the induction step (step 2 in the refinement strategy above) satisfies those conditions. We start with the case $m=2$.

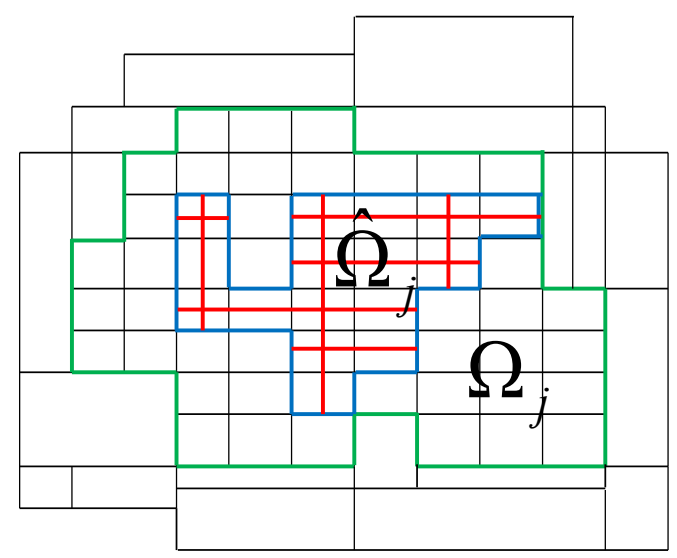

Figure 13: The boundary of $\Omega_{j}$ is shown in green; the boundary of $\widehat{\Omega}_{j}$ is blue; and the line-segments $E_{j 1}, \ldots, E_{j n_{j}}$ are red. (For interpretation of the references to color in this figure legend, the reader is referred to the web version of this article.)

\subsection{The case $m=2$}

Let $T=T_{N}$ be a $T$-mesh of type 2 for some integer $N \geqslant 1$ (see Definition 8). We consider the induction step from $T_{j}$ to $T_{j+1}$, for a given $0 \leqslant j \leqslant N-1$, such that $T_{j+1}$ is derived from $T_{j}$ by refining with maximal interior segments $E_{j 1}, \ldots, E_{j n_{j}}$, in compliance with step 2 of the algorithm in Definition 8. For a given order of the line-segments $E_{j 1} \prec \cdots \prec E_{j n_{j}}$, the T-mesh $T_{j i}, 1 \leqslant$ $i \leqslant n_{j}$ is derived from $T_{j}$ by successive introduction of the line-segments $E_{j 1}, \ldots, E_{j i}$; in particular, $T_{j n_{j}}=T_{j+1}$.

In the following lemma we omit the index $j$ to simplify the notations, observing that the proof is the same for any $0 \leqslant j \leqslant N-1$; thus $T_{i}$ corresponds to $T_{j i}, E_{i}$ to $E_{j i}, \widehat{\Omega}$ and $\Omega$ to $\widehat{\Omega}_{j}$ and $\Omega_{j}$ respectively. In formulating 
the basis functions, we will presume that the cells of the domain $\Omega$ are of the same size and any line-segment that divides a cell of the domain $\widehat{\Omega}$ connects the mid-points of opposite edges of that cell.

Lemma 3. There exists an ordered sequence of maximal interior segments $E_{1} \prec \cdots \prec E_{n}$ which refine $T_{0}$ such that the projections $P_{E_{i}}: W\left[T_{i}\right] \rightarrow W\left[E_{i}\right]$ are surjective for all $i=1 \ldots n$, so for any $i=1 \ldots n$ there is a set of spline functions $\left\{f_{i 1}, \ldots f_{i l_{i}}\right\} \subset S(2,2,1,1)\left(T_{i}\right)$, such that $\left\{P_{E_{i}}\left(K_{i}\left(f_{i 1}\right)\right), \ldots\right.$, $\left.P_{E_{i}}\left(K_{i}\left(f_{i l_{i}}\right)\right)\right\}$ forms a basis of $W\left[E_{i}\right]$, where $K_{i}: S(2,2,1,1)\left(T_{i}\right) \rightarrow W\left[T_{i}\right]$ is given in (12). Moreover, these spline functions can be chosen such that their supports are contained within $\Omega$.

Proof. The proof could be found in Section 5 .

Remark 2. Wu et al. [22] describe a special classes $\mathcal{T}_{m, m}$ of hierarchical Tmeshes. If $m=2$, then $\mathcal{T}_{2,2}$ is the ordinary class of crosswise hierarchical $T$ meshes with a level structure, which is the same to one used for PHT-splines [16]. The following procedure, shown in Fig.14, can be used to generate a Tmesh of class $\mathcal{T}_{2,2}$. We begin with a tensor-product mesh over a rectangular domain (the cells of this mesh are defined to be of level 0). In order to generate a cell at level $j+1$, a cell at level $j$ must be subdivided into four subcells (which are defined to be at level $j+1$ ). A cell is divided by two line-segments connecting the mid-points of opposite edges.

We observe that a hierarchical $T$-mesh of class $\mathcal{T}_{2,2}$ is of type 2 , following Definition 8. Let $T$ be a hierarchical T-mesh of class $\mathcal{T}_{2,2}$. The domain $\widehat{\Omega}_{j}$, which is to be divided to obtain cells at level $j+1$, is composed of cells at level $j$. We create a domain $\Omega_{j} \supset \widehat{\Omega}_{j}$ by unifying all the cells at level $j$. If $j \geqslant 1$, then the construction of $T$ means that $\Omega_{j}$ is composed of $2 \times 2$ blocks of cells at level $j$ (each corresponding to a single cell at level $j-1$ ). In order to prove that $\Omega_{j}$ admits an inner offset at a distance $\frac{1}{2}$, we need to show that $\mathbb{R}^{2} \backslash \Omega_{j}$ admits an offset at a distance $\frac{1}{2}$. The domain $\mathbb{R}^{2} \backslash \Omega_{j}$ is also composed of $2 \times 2$ blocks of cells. It is apparent that the cells from the offset region $R_{1}$ of a domain $\mathbb{R}^{2} \backslash \Omega_{j}$ are related to $\mathbb{R}^{2} \backslash \Omega_{j}$ through the connections shown in Fig.5. This observation implies that $\mathbb{R}^{2} \backslash \Omega_{j}$ admits an offset at a distance $\frac{1}{2}$. If $j=0$, then it suffices to suppose that the original tensor-product mesh extends at least 2 cells in both coordinate directions to guarantee that $\Omega_{0}$ admits an inner offset at a distance $\frac{1}{2}$. We assume that the cells at level 0 having common edges with the boundary of $T$ are not divided, i.e. starting at level 1, all introduced line-segments will be maximal 
interior segments of $T$. Thus, by Definition 8, $T$ is of type 2. The spline basis functions of $S(2,2,1,1)(T)$ can be obtained by Lemma 3.
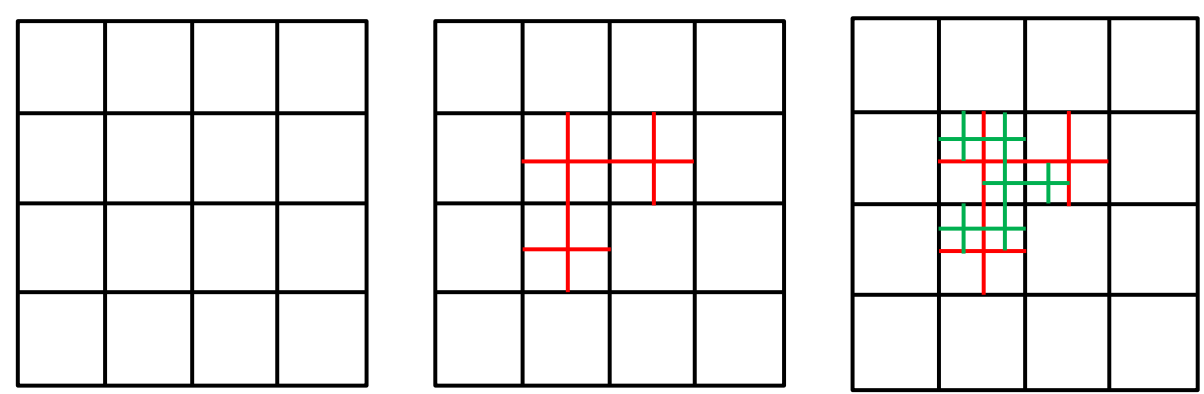

Figure 14: Generation of a hierarchical T-mesh of class $\mathcal{T}_{2,2}$ starts with a tensor-product mesh over a rectangular domain (left); 3 cells at level 0 are subdivided by the red linesegments into 12 cells at level 1 (center); 6 cells at level 1 are subdivided by the green line-segments into 24 cells at level 2 (right). (For interpretation of the references to color in this figure legend, the reader is referred to the web version of this article.)

\subsection{The case $m=3$}

Let $T=T_{N}$ be a T-mesh of type 3 for some integer $N \geqslant 1$ (see Definition 8). As we did in the previous subsection, we will consider the induction step from $T_{j}$ to $T_{j+1}$, for a given $0 \leqslant j \leqslant N-1$, which allows us to derive $T_{j+1}$ from $T_{j}$ by refining the $\mathrm{T}$-mesh $T_{j}$ by introducing maximal interior segments $E_{j 1}, \ldots, E_{j n_{j}}$, as we did in step 2 of the algorithm in Definition 8. For a given ordering of the line-segments $E_{j 1} \prec \cdots \prec E_{j n_{j}}$, the T-mesh $T_{j i}, 1 \leqslant i \leqslant n_{j}$ can be derived from $T_{j}$ through the consistent refinement by the line-segments $E_{j 1}, \ldots, E_{j i}$; in particular, $T_{j n_{j}}=T_{j+1}$.

In the following lemma we will omit the index $j$. In addition, we will suppose that the cells of the domain $\Omega$ are of the same size and any linesegment that divides a cell of the domain $\widehat{\Omega}$ connects the mid-points of opposite edges of that cell, as we did in Lemma 3.

Lemma 4. There exists an ordered sequence of maximal interior segments $E_{1} \prec \cdots \prec E_{n}$ which refine $T_{0}$ such that the projections $P_{E_{i}}: W\left[T_{i}\right] \rightarrow W\left[E_{i}\right]$ are surjective for all $i=1 \ldots n$, so for any $i=1 \ldots n$ there $i s$ a set of spline functions $\left\{f_{i 1}, \ldots f_{i l_{i}}\right\} \subset S(3,3,2,2)\left(T_{i}\right)$ such that $\left\{P_{E_{i}}\left(K_{i}\left(f_{i 1}\right)\right), \ldots\right.$, $\left.P_{E_{i}}\left(K_{i}\left(f_{i l_{i}}\right)\right)\right\}$ forms a basis of $W\left[E_{i}\right]$, where $K_{i}: S(3,3,2,2)\left(T_{i}\right) \rightarrow W\left[T_{i}\right]$ is given in (12). Moreover, these spline functions can be chosen such that their supports are contained within $\Omega$. 
Proof. The proof could be found in Section 6 .

Remark 3. Let us briefly revisit the construction of $T$-meshes of class $\mathcal{T}_{3,3}$, as described by Wu et al. [22], and illustrated in Fig.15. Their procedure starts with a tensor-product mesh over a rectangular domain (containing cells that are defined to be at level 0). In order to generate a cell at level $j+1$, one has to subdivide a quad of four neighboring cells at level $j$ into sixteen subcells (which are defined to be at level $j+1$ ). Any two $2 \times 2$ blocks of cells at level $j$ which are to be subdivided must either have two common boundary edges, one common boundary vertex, or no common vertices. It must also be assumed that each cell is subdivided by two straight lines connecting the mid-points of the two opposite edges.
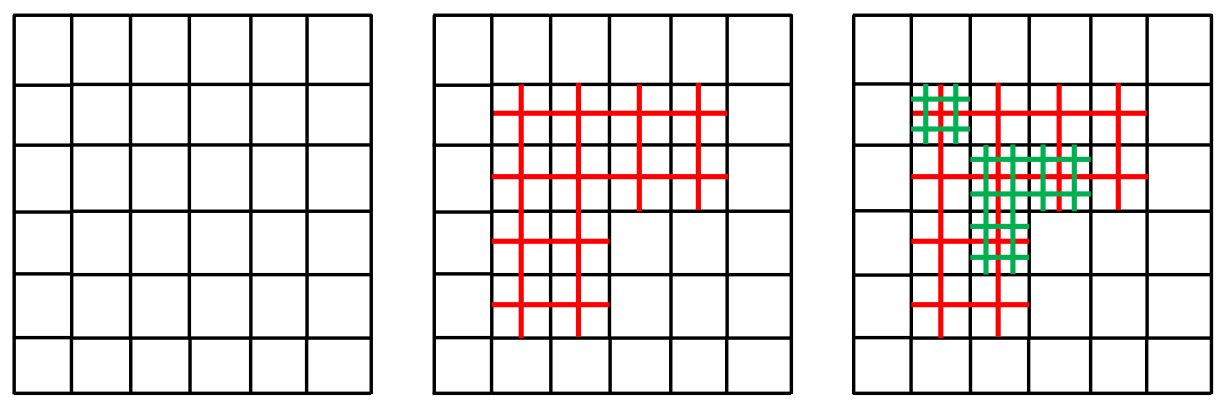

Figure 15: The generation of a hierarchical T-mesh of class $\mathcal{T}_{3,3}$ starts with a tensorproduct mesh over a rectangular domain (left); 3 quads of cells at level 0 are subdivided by the red line-segments into 48 cells at level 1 (center); 4 quads of cells at level 1 are subdivided by the green line-segments into 64 cells at level 2 (right). (For interpretation of the references to color in this figure legend, the reader is referred to the web version of this article.)

We see from Definition 8 that a hierarchical $T$-mesh of class $\mathcal{T}_{3,3}$ is of type 3. Let $T$ be a hierarchical T-mesh of class $\mathcal{T}_{3,3}$. The domain $\widehat{\Omega}_{j}$, which is to be divided to obtain cells at level $j+1$, is composed of $2 \times 2$ blocks of cells at level $j$. We can create a domain $\Omega_{j} \supset \widehat{\Omega}_{j}$ by unifying all the cells at level $j$. If $j \geqslant 1$, then, because of the way in which $T$ was constructed, $\Omega_{j}$ is composed of $4 \times 4$ blocks at level $j$ (originally the $2 \times 2$ blocks at level $j-1$ ). In order to prove that $\Omega_{j}$ admits an inner offset at a distance 1 , we need to show that $\mathbb{R}^{2} \backslash \Omega_{j}$ admits an offset at a distance 1 . The domain $\mathbb{R}^{2} \backslash \Omega_{j}$ is also composed of $4 \times 4$ blocks of cells. It is apparent that the cells from the offset region $R_{1}$ of a domain $\mathbb{R}^{2} \backslash \Omega_{j}$ are related to $\mathbb{R}^{2} \backslash \Omega_{j}$ through the connections 
shown in Fig.6. This observation implies that $\mathbb{R}^{2} \backslash \Omega_{j}$ admits an offset at a distance 1 . If $j=0$, then it suffices to suppose that the original tensorproduct mesh has at least 3 cells in both coordinate directions to guarantee that $\Omega_{0}$ admits an inner offset at a distance 1 . We assume that the cells at level 0 having common edges with the boundary of $T$ are not divided, i.e. starting at level 1, all introduced line-segments will be maximal interior segments of T. Thus, by Definition 8, T is of type 3. From Lemma 4 we have that for the T-mesh $T$, the line-segments, which are used to divide the cells of level $j$, may be arranged in the following simple way. The line-segments of length $\geqslant 4$ need not be ordered. Then, the remaining line-segments, which are of length 2, may also be ordered arbitrarily, except for isolated quads (see Fig.22, right), which can simply be ordered in a clockwise direction. The spline basis functions of $S(3,3,2,2)(T)$ can be obtained by Lemma 4 .

\subsection{Some necessary remarks on a modification of the refinement strategy}

From the proof of Lemma 4, we see that spline basis functions can be obtained; but, for example, this may involve the sophisticated construction shown in Fig. 21 (center). It is more practical to use configurations in which the line-segments can be ordered in a simple or even arbitrary way. From this point of view, it is reasonable to assume that all maximal interior segments are of length $\geqslant m$. This latter assumption ensures us that the corresponding $\mathrm{T}$-mesh is regular, defined as in Mourrain [25]; in particular, it satisfies the conditions of Lemma 2 and the dimension $\operatorname{dim} S(m, m, m-1, m-1)(T)$ can be obtained trough the formula (15).

This assumption enables us to order the line-segments within a step 2 of the refinement strategy in arbitrary way. Moreover, it enables us not to assume that the line-segments $E_{j 1}, \ldots, E_{j n_{j}}$ refining $T_{j}$ have endpoints on $\partial \widehat{\Omega}_{j}$. In addition, we do not need to assume that each cell of $\widehat{\Omega}_{j}$ is divided following the rule illustrated in Fig.12; we may admit that a cell of $\widehat{\Omega}_{j}$ is divided by several line-segments in both directions. A step 2 of this modified refinement strategy is illustrated in Fig.16. From the proofs of Lemmas 3 and 4 we see that the spline basis functions are tensor-product B-splines except probably those which correspond to the line-segments intersecting in vertices that are close enough to the boundary $\partial \Omega_{j}$. In order to guarantee that all the spline basis functions are tensor-product B-splines, we need to extend the line-segments intersecting in each vertex $Z$, for which $f_{Z}$ cannot be represented as a tensor-product B-spline, until the boundary of a domain $\Omega_{Z}$ (see eg. Fig.20). 


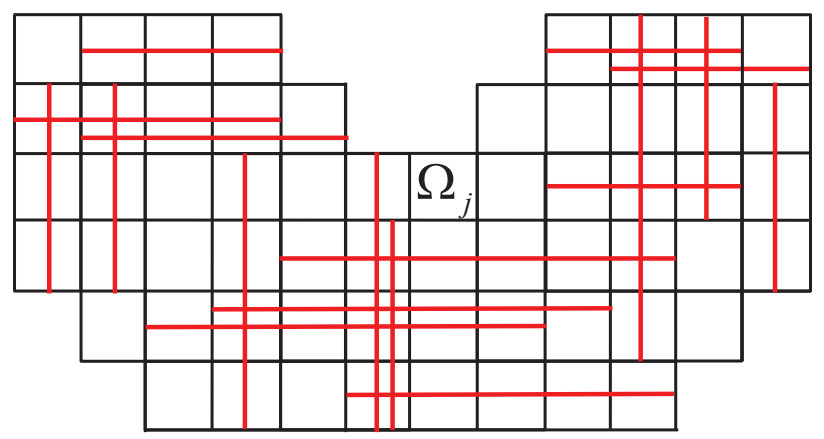

Figure 16: The domain $\widehat{\Omega}_{j}$ is composed of the black cells crossed by red line-segments. The domain $\Omega_{j}$ is composed of all the black cells. This configuration is suitable for $m=2$ and $m=3$, because $\Omega_{j}$ admits an inner offset at a distance 1 and all red line-segments are of length $\geqslant 3$. (For interpretation of the references to color in this figure legend, the reader is referred to the web version of this article.)

\section{Conclusions}

We have described an iterative strategy for generating consistent hierarchical $\mathrm{T}$-meshes. It is based on the concept of a domain of restricted configuration, which is composed of the cells of a tensor-product mesh, and which is divided in successive steps. We have described the required ordering of line-segments during refinement, and the construction of basis functions for splines of degree 2 and 3.

We have shown that hierarchical $\mathrm{T}$-meshes of classes $\mathcal{T}_{2,2}$ and $\mathcal{T}_{3,3}$ are produced by this strategy for degrees 2 and 3 respectively. We have described the relevant modifications of our strategy which substantially simplify the ordering of line-segments and the corresponding construction of basis functions.

The concept of consistent hierarchical T-meshes is promising for applications as long as they allow local refinement and offer a simple way for describing spline basis functions. Our iterative strategy provides feasible framework for generating consistent hierarchical $\mathrm{T}$-meshes. We believe that the results of this paper might help in selection and processing of possible implementations of consistent hierarchical $\mathrm{T}$-meshes. In our future work we intend to propose analogues of consistent hierarchical $\mathrm{T}$-meshes for trivariate case and offer a strategy for generating of such meshes. 


\section{Appendix A}

In this appendix we will give the proof of Lemma 3.

Proof. Given a maximal interior segment $E \in\left\{E_{1}, \ldots, E_{n}\right\}$, let $L(E)$ denote the number of cells of $\widehat{\Omega} \subset \Omega$ that are divided by the line-segment $E$; we will call $L(E)$ the length of $E$. An admissible ordering of the linesegments $E_{1}, \ldots, E_{n}$ can be obtained quite simply by ensuring that all the line-segments of length $\geqslant 2$ precede those of length 1 .

However, this issue is more easily understood if we arrange the linesegments $E_{1}, \ldots, E_{n}$ as follows. Let $E_{1} \prec \cdots \prec E_{k_{1}}$ be all the vertical line-segments of length $\geqslant 2$, ordered from left to right; similarly let $E_{k_{1}+1} \prec$ $\ldots \prec E_{k_{2}}$ be all the horizontal line-segments of length $\geqslant 2$, ordered from bottom to top. The remaining line-segments $E_{k_{2}+1} \prec \cdots \prec E_{n}$ of length 1 do not need to be ordered.

Since $\Omega$ admits an inner offset at a distance $\frac{1}{2}$, the lemma statement above applies to the line-segments $E_{1} \prec \cdots \prec E_{k_{1}}$ as a direct consequence of Proposition 2.

For a given horizontal line-segment $E_{i}, k_{1}+1 \leqslant i \leqslant k_{2}$, let $Z$ be the location of the intersection with a vertical line-segment $E_{j}, j \leqslant k_{1}$. Consider two short subsegments $H$ from $E_{i}$ and $V$ from $E_{j}$ length 2, which intersect at $Z$; such subsegments always exist because $L\left(E_{i}\right), L\left(E_{j}\right) \geqslant 2$. Since $\Omega$ admits

an inner offset at a distance $\frac{1}{2}$, one of the two configurations of cells shown in Fig. 17 must occur.
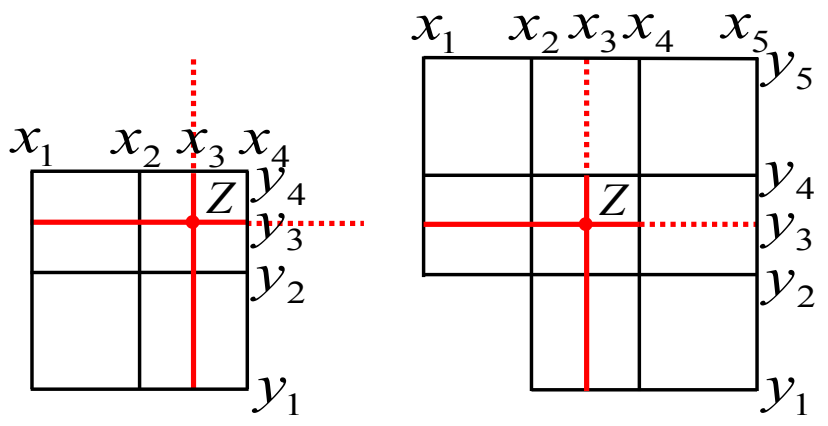

Figure 17: The subsegments $H$ and $V$ are drawn in red, and their possible extensions are shown dashed; the black cells belong to the domain $\Omega$. In both the left and right configurations, the $H$ has the endpoints $\left(x_{1}, y_{3}\right)$ and $\left(x_{4}, y_{3}\right)$, and the endpoints of $V$ are $\left(x_{3}, y_{1}\right)$ and $\left(x_{3}, y_{4}\right)$. (For interpretation of the references to color in this figure legend, the reader is referred to the web version of this article.) 
We can now obtain a spline function $f_{Z}(x, y) \in S(2,2,1,1)\left(T_{i}\right)$, using one of two formulas, depending on which of the configurations shown in Fig.17 actually occurs. For the first configuration, on the left in Fig.17, the function $f_{Z}(x, y)$ is defined as follows:

$$
f_{Z}(x, y)=N\left[x_{1}, x_{2}, x_{3}, x_{4}\right](x) N\left[y_{1}, y_{2}, y_{3}, y_{4}\right](y) ;
$$

and for the second configuration, on the right in Fig.17, as follows:

$$
\begin{aligned}
& f_{Z}(x, y)=N\left[x_{1}, x_{2}, x_{3}, x_{4}\right](x) N\left[y_{2}, y_{3}, y_{4}, y_{5}\right](y)+ \\
& N\left[x_{2}, x_{3}, x_{4}, x_{5}\right](x) N\left[y_{2}, y_{3}, y_{4}, y_{5}\right](y)+ \\
& N\left[x_{2}, x_{3}, x_{4}, x_{5}\right](x) N\left[y_{1}, y_{2}, y_{3}, y_{4}\right](y) \text {. }
\end{aligned}
$$

This latter function is defined in the space $S(2,2,1,1)\left(T_{i}\right)$, because the functions:

$$
\begin{gathered}
N\left[x_{1}, x_{2}, x_{3}, x_{4}\right](x)+N\left[x_{2}, x_{3}, x_{4}, x_{5}\right](x), \\
N\left[y_{1}, y_{2}, y_{3}, y_{4}\right](y)+N\left[y_{2}, y_{3}, y_{4}, y_{5}\right](y)
\end{gathered}
$$

are all of class $C^{\infty}$ at the points $x_{3}$ and $y_{3}$, with respect to $x$ and $y$ respectively. The conformality factor $k_{Z}$ for the vertex $Z$, corresponding to the function $f_{Z}(x, y)$, is nonzero. Indeed, proving this by contradiction, if we suppose that $k_{Z}=0$, then the conformality factors for the vertices on the line-segments $H$ and $V$ are zero (because only three knots remain on $H$ and three on $V$ ), and the function $f_{Z}(x, y)$ is of class $C^{\infty}$ with respect to $x$ and $y$ along the line-segments $V$ and $H$ respectively using similar reasoning to Lemma 1, which contradicts formulas (17) and (18).

Let $U_{i}$ denote the set of vertices in line-segment $E_{i}$, such that each element of $U_{i}$ is an intersection of $E_{i}$ with a vertical line-segment $E_{j}, j \leqslant k_{1}$. Thus, for any vertex $Z \in U_{i}$, there is a spline function $f_{Z} \in S(2,2,1,1)\left(T_{i}\right)$, such that the corresponding conformality factor $k_{Z} \neq 0$; and $k_{\widehat{Z}}=0$ for any other vertex $\widehat{Z} \in U_{i}, \widehat{Z} \neq Z$. Let $\widehat{E}_{i}$ denote the line-segment which is obtained from $E_{i}$ by eliminating vertices belonging to the set $U_{i}$. By virtue of Proposition 2, there are $s_{i}=L\left(E_{i}\right)-m$ tensor-product B-splines $\left\{f_{i 1}, \ldots, f_{i s_{i}}\right\} \subset S(2,2,1,1)\left(T_{i}\right)$ with supports within the domain $\Omega$ such that the set $\left\{P_{\widehat{E}_{i}}\left(K_{i}\left(f_{i 1}\right)\right), \ldots, P_{\widehat{E}_{i}}\left(K_{i}\left(f_{i s_{i}}\right)\right)\right\}\left(\right.$ where $K_{i}: S(2,2,1,1)\left(T_{i}\right) \rightarrow W\left[T_{i}\right]$ is defined by (12)) forms a basis of the space $W\left[\widehat{E}_{i}\right]$. Thus, there are $l_{i}=s_{i}+\# U_{i}$ spline functions $\bigcup_{j=1}^{s_{i}} f_{i j} \cup \bigcup_{Z \in U_{i}} f_{Z} \subset S(2,2,1,1)\left(T_{i}\right)$ which have 
projections under the map $P_{E_{i}} \circ K_{i}$ that form the basis of the space $W\left[E_{i}\right]$; moreover, because of the way they are constructed, these functions have supports within the domain $\Omega$. There is nothing to prove for the remained line-segments $E_{k_{2}+1} \prec \cdots \prec E_{n}$, because $W\left[E_{i}\right]=\mathbf{0}$ for any $k_{2}+1 \leqslant i \leqslant n$. Thus, Lemma 3 is proved.

\section{Appendix B}

In this appendix we will give the proof of Lemma 4 .

Proof. As we did in the proof of Lemma 3, we arrange the line-segments $E_{1} \prec \ldots \prec E_{n}$ in order of their lengths $L\left(E_{i}\right), i=1 \ldots n$. Line-segments of length $\geqslant 3$ can be arranged in an arbitrary way; but we will suppose that $E_{1} \prec \cdots \prec E_{k_{1}}$ are all the vertical line-segments of length $\geqslant 3$, arranged from left to right. Similarly, $E_{k_{1}+1} \prec \cdots \prec E_{k_{2}}$ are all the horizontal linesegments of length $\geqslant 3$, arranged from bottom to top. The required ordering for line-segments of length $\leqslant 2$ will be specified later.

Since $\Omega$ admits an inner offset at a distance 1, the lemma statement above applies to the line-segments $E_{1} \prec \cdots \prec E_{k_{1}}$ as a direct consequence of Proposition 2.

Let $Z$ be the vertex at which a horizontal line-segment $E_{i}, k_{1}+1 \leqslant i \leqslant k_{2}$ intersects with a vertical line-segment $E_{j}$, which has index $j \leqslant k_{1}$. We will suppose that $Z$ is not inside the leftmost or the rightmost cell divided by the horizontal line-segment $E_{i}$. Consider two short subsegments, $H$ from $E_{i}$ and $V$ from $E_{j}$, both of length 3 , which intersect at $Z$, such that the cell that contain $Z$ is the middle cell divided by $H$.

Let $\Omega_{Z}$ be the possible minimal domain composed of cells from domain $\Omega$, which contains subsegments $H$ and $V$, and admits an inner offset at a distance 1. The possible configurations of the domain $\Omega_{Z}$ are shown in Fig.18 and 20 (left). For spline function $f_{Z}$ corresponding to the configuration in Fig.20 (left) is:

$$
\begin{aligned}
f_{Z}(x, y)= & N\left[x_{1}, x_{2}, x_{3}, x_{4}, x_{5}\right](x) N\left[y_{2}, y_{3}, y_{4}, y_{5}, y_{6}\right](y)+ \\
& \frac{3}{5} N\left[x_{2}, x_{3}, x_{4}, x_{5}, x_{6}\right](x) N\left[y_{2}, y_{3}, y_{4}, y_{5}, y_{6}\right](y)+ \\
& \frac{3}{5} N\left[x_{1}, x_{2}, x_{3}, x_{4}, x_{5}\right](x) N\left[y_{1}, y_{2}, y_{3}, y_{4}, y_{5}\right](y) .
\end{aligned}
$$

This function is defined in the space $S(3,3,2,2)\left(T_{i}\right)$ because functions:

$$
N\left[x_{1}, x_{2}, x_{3}, x_{4}, x_{5}\right](x)+\frac{3}{5} N\left[x_{2}, x_{3}, x_{4}, x_{5}, x_{6}\right](x),
$$




$$
\frac{3}{5} N\left[y_{1}, y_{2}, y_{3}, y_{4}, y_{5}\right](y)+N\left[y_{2}, y_{3}, y_{4}, y_{5}, y_{6}\right](y)
$$

are all of class $C^{\infty}$ at the points $x_{4}$ and $y_{3}$, with respect to $x$ and $y$ respectively.

We will now suppose that the vertex $Z$ is inside either the leftmost or the rightmost cell divided by the horizontal line-segment $E_{i}$. Again $H$ and $V$ are subsegments, both of length 3 , of $E_{i}$ and $E_{j}$ respectively, which intersect at $Z$. The possible configurations of the domain $\Omega_{Z}$ are shown in the Fig.19 and Fig.20 (right). The spline function $f_{Z}$ corresponding to the configuration in the Fig.20 (right) is:

$$
\begin{aligned}
f_{Z}(x, y)= & -N\left[x_{1}, x_{2}, x_{3}, x_{4}, x_{5}\right](x) N\left[y_{3}, y_{4}, y_{5}, y_{6}, y_{7}\right](y)+ \\
+ & N\left[x_{3}, x_{4}, x_{5}, x_{6}, x_{7}\right](x) N\left[y_{3}, y_{4}, y_{5}, y_{6}, y_{7}\right](y)+ \\
& +N\left[x_{1}, x_{2}, x_{3}, x_{4}, x_{5}\right](x) N\left[y_{1}, y_{2}, y_{3}, y_{4}, y_{5}\right](y)
\end{aligned}
$$

This function is in the space $S(3,3,2,2)\left(T_{i}\right)$ because the functions

$$
\begin{gathered}
N\left[x_{1}, x_{2}, x_{3}, x_{4}, x_{5}\right](x)-N\left[x_{3}, x_{4}, x_{5}, x_{6}, x_{7}\right](x), \\
N\left[y_{1}, y_{2}, y_{3}, y_{4}, y_{5}\right](y)-N\left[y_{3}, y_{4}, y_{5}, y_{6}, y_{7}\right](y)
\end{gathered}
$$

are all of class $C^{\infty}$ at the points $x_{4}$ and $y_{4}$, with respect to $x$ and $y$ respectively.

For the sake of brevity, we will not provide formulas for the spline function $f_{Z} \in S(3,3,2,2)\left(T_{i}\right)$ for all possible configurations of the domain $\Omega_{Z}$ (these configurations are shown in Figs.18 and 19). However, we will prove that the function $f_{Z}$ exists by means of the following simple consideration. We extend the subsegments $H$ and $V$ inside $\Omega_{Z}$ until they meet the boundary $\partial \Omega_{Z}$; let $\widetilde{H}$ and $\widetilde{V}$ denote the extended line-segments. Further, let $R^{\prime}$ denote some tensor-product mesh over a rectangular domain, such that each cell of $\Omega_{Z}$ is a cell of $R^{\prime}$, and the boundary $\partial \Omega_{Z}$ has no common edges with the boundary of $R^{\prime}$. Applying Lemma 2 to the T-mesh $R=R^{\prime} \cup H \cup V$, we find that $\operatorname{dim} S(3,3,2,2)(R)=\operatorname{dim} S(3,3,2,2)\left(R^{\prime}\right)+1$ (Deploying Lemma 2 to deal with such a simple case is like using a steam-hammer to crack a nut; but it wide come into its own when we arrive at a more complicated construction shortly).

Let $\widetilde{R}$ denote the T-mesh $R^{\prime} \cup \widetilde{H} \cup \widetilde{V}$. Applying Proposition 1 and 2 to refine $R^{\prime}$ using the subsegments $\widetilde{H}$ and $\widetilde{V}$, we obtain $S(3,3,2,2)(\widetilde{R})$ $=S(3,3,2,2)\left(R^{\prime}\right) \bigoplus \operatorname{span}\left\{f_{1}, \ldots, f_{l}\right\}$, where $f_{1}, \ldots, f_{l}$ are tensor-product 
B-splines that have supports within the domain $\Omega_{Z}$. Since $S(3,3,2,2)\left(R^{\prime}\right)$ $\subset S(3,3,2,2)(R) \subset S(3,3,2,2)(\widetilde{R})$, an appropriate spline function $f_{Z} \in$ $S(3,3,2,2)(R)$ (such that $\left.S(3,3,2,2)(R)=S(3,3,2,2)\left(R^{\prime}\right) \bigoplus \operatorname{span}\left\{f_{Z}\right\}\right)$ can be obtained by taking a linear combination of $f_{1}, \ldots, f_{l}$, which will have support within the domain $\Omega_{Z}$. Thus the existence of a function $f_{Z}$ for the subsegments $H \subset E_{i}, V \subset E_{j}$ and $H \cap V=\{Z\}$ is proved. By exactly the same arguments as we used in Lemma 3 , we extend this lemma to all the line-segments $E_{k_{1}+1} \prec \cdots \prec E_{k_{2}}$.
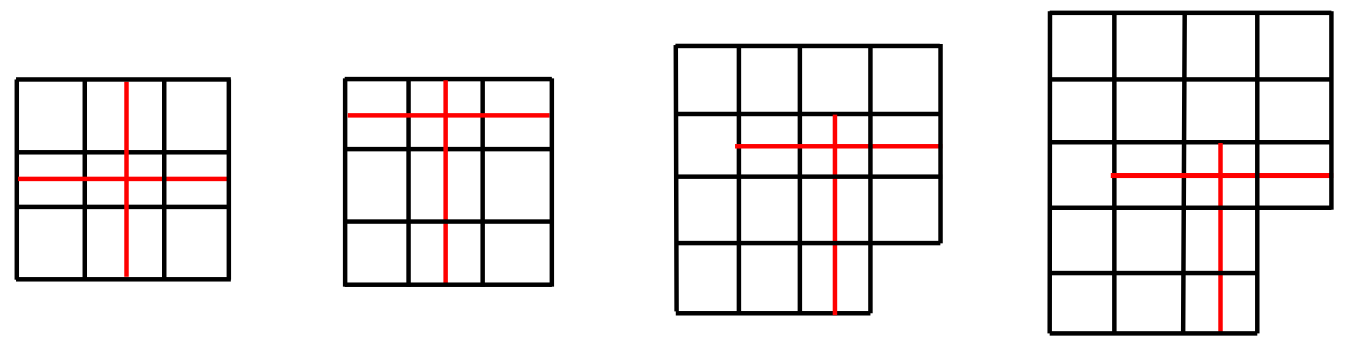

Figure 18: The black cells form the domain $\Omega_{Z} \subset \Omega$; subsegments $H$ and $V$ are shown in red. (For interpretation of the references to color in this figure legend, the reader is referred to the web version of this article.)
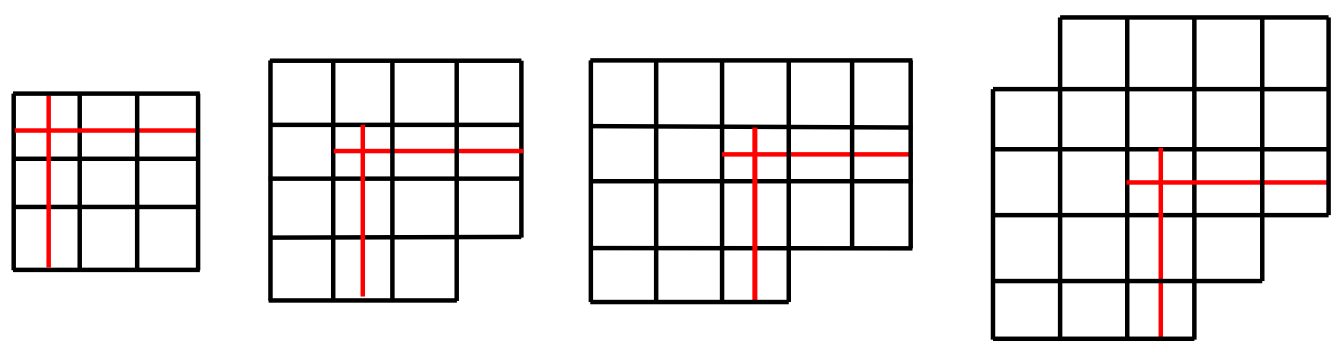

Figure 19: The black cells form the domain $\Omega_{Z} \subset \Omega$; subsegments $H$ and $V$ are shown in red. (For interpretation of the references to color in this figure legend, the reader is referred to the web version of this article.)

We can now see how to order the line-segments $E_{k_{2}+1} \prec \cdots \prec E_{k_{3}}$ of length 2 , from the following observation. Since the line-segments $E_{1}, \ldots, E_{n}$ refining $T_{0}$ have endpoints on $\partial \widehat{\Omega}$, the set of line-segments of length 2 can be decomposed into chains of three types. A chain of the first type connects two (possibly identical) line-segments of length $\geqslant 3$ (see Fig.21). A chain of the second type intersects with a line-segment of length $\geqslant 3$ at just one vertex 

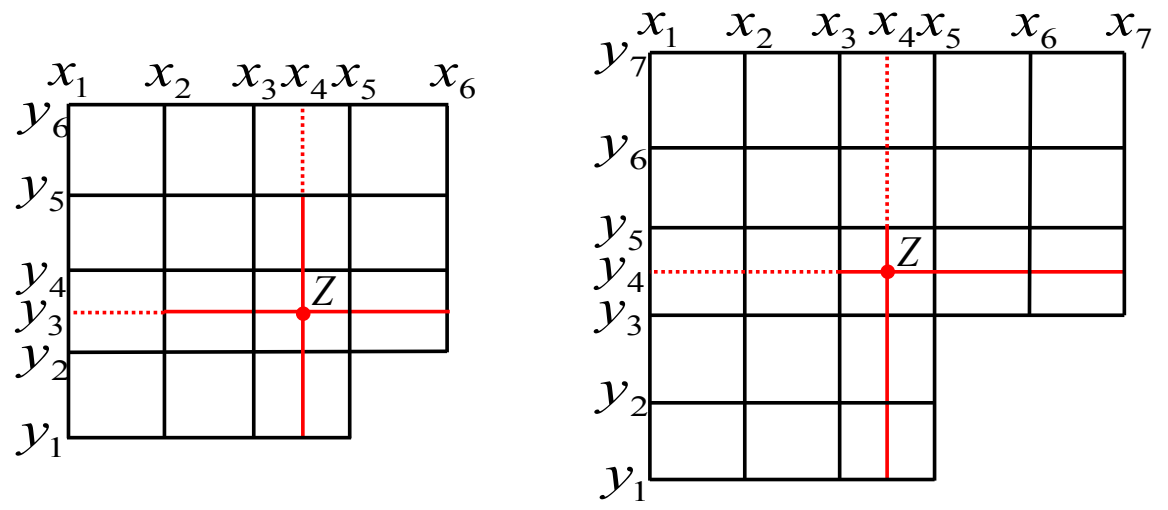

Figure 20: The black cells form the domain $\Omega_{Z} \subset \Omega$; subsegments $H$ and $V$ are shown in red with possible extensions shown as dashed lines. (For interpretation of the references to color in this figure legend, the reader is referred to the web version of this article.)
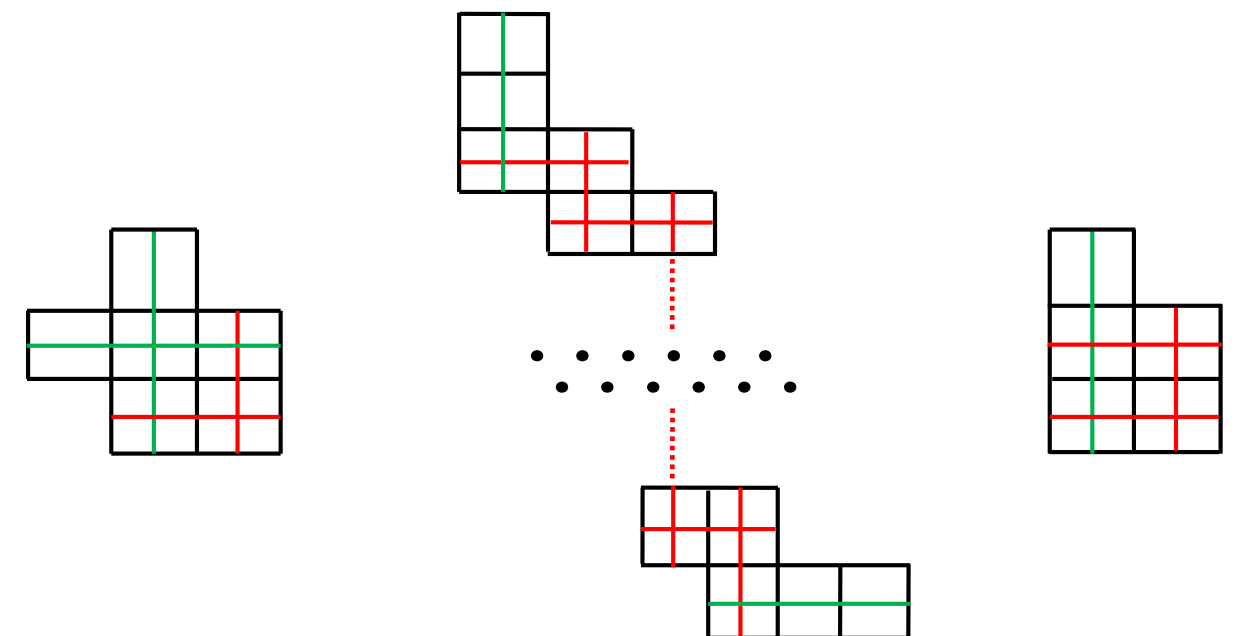

Figure 21: Organizing line-segments into chains of the first type; line-segments of length 2 , forming each chain, are shown in red; line-segments of length $\geqslant 3$ are shown in green; the case of connected line-segments of length $\geqslant 3$ that coincide is shown on the right. (For interpretation of the references to color in this figure legend, the reader is referred to the web version of this article.) 


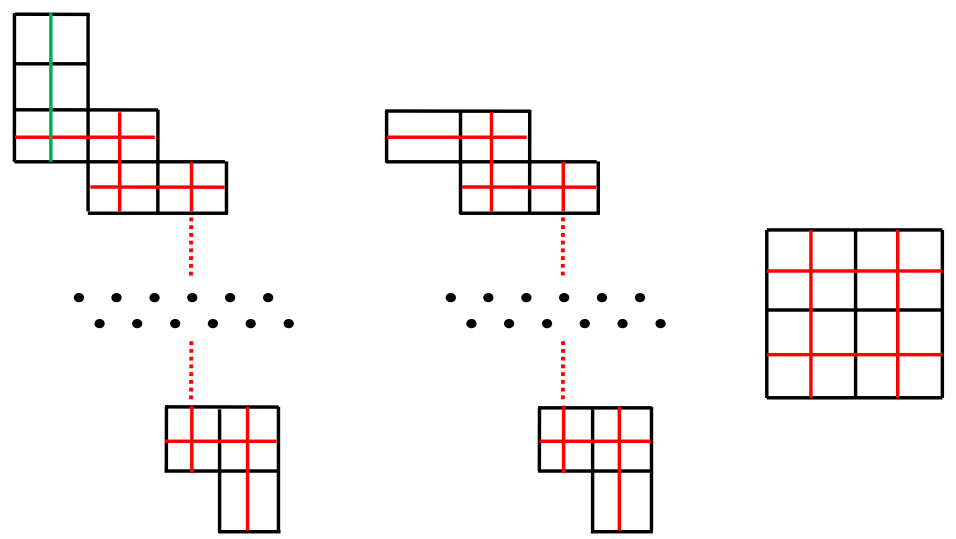

Figure 22: A chain of the second type (left); a chain of the third type (center); and a special case of a chain of the third type (right). The line-segments, forming each chain, are shown in red. (For interpretation of the references to color in this figure legend, the reader is referred to the web version of this article.)

(see Fig.22, left). A chain of the third type has no intersections with any line-segment of length $\geqslant 3$ (see Fig.22, center); in a special case a chain of the third type divides four neighboring cells, as shown in Fig.22 (right). The set of chains does not need to be organized, but the line-segments in each chain must be ordered: for example in Fig.21 (center) and Fig.22 (left and center) the line-segments might be ranged from upper-left to lower-right; and in Fig.22 (right) the line-segments might run clockwise. From Lemma 2, only a chain of the first type (see Fig.21) and the type of chain shown in Fig.22 (right) provide a nontrivial spline function. From Propositions 1 and 2 we see that this function can be chosen with a support inside the domain $\Omega$. There is nothing to prove for the remaining line-segments $E_{k_{3}+1} \prec \cdots \prec E_{n}$ of length 1 , because $W\left[E_{i}\right]=\mathbf{0}$ for any $k_{3}+1 \leqslant i \leqslant n$. Lemma 4 is proved. $\square$

\section{Acknowledgment}

This research was supported by the National Research Foundation of Korea (NRF) grant funded by the Korea government (MEST)(No. 201200005043) and partially funded by the Ministry of Knowledge Economy (MKE, Korea) (No. 10035474). Durkbin Cho was supported by Basic Science Research Program through the National Research Foundation of Korea (NRF) funded by the Ministry of Science, ICT \& Future Planning (No. 2012R1A1A1006109). 


\section{References}

[1] T. J. R. Hughes, J. A. Cottrell, Y. Bazilevs, Isogeometric analysis: CAD, finite elements, NURBS, exact geometry, and mesh refinement, Computer Methods in Applied Mechanics and Engineerinig 194 (2005) 41354195.

[2] J. A. Cottrell, T. J. R. Hughes, Y. Bazilevs, Isogeometric Analysis: Toward Integration of CAD and FEA, John Wiley \& Sons, 2009.

[3] T. W. Sederberg, J. Zheng, A. Bakenov, A. Nasri, T-splines and TNURCCS, ACM Transactions on Graphics 22 (2003) 477-484.

[4] T. W. Sederberg, D. L. Cardon, G. T. Finnigan, N. S. North, J. Zheng, T. Lyche, T-splines simplification and local refinement, ACM Transactions on Graphics 23 (2004) 276-283.

[5] A. Buffa, D. Cho, G. Sangalli, Linear independence of the T-spline blending functions associated with some particular T-meshes, Computer Methods in Applied Mechanics and Engineerinig 199 (2010) 1437-1445.

[6] Y. Bazilevs, V. M. Calo, J. A. Cottrell, J. A. Evans, T. J. R. Hughes, S. Lipton, M. A. Scott, T. W. Sederberg, Isogeometric analysis using tsplines, Computer Methods in Applied Mechanics and Engineerinig 199 (2010) 229-263.

[7] X. Li, J. Zheng, T. W. Sederberg, T. J. R. Hughes, M. A. Scott, On linear independence of the T-spline blending functions, Computer Aided Geometric Design 29 (1) (2012) 63-76.

[8] M. A. Scott, X. Li, T. W. Sederberg, T. J. R. Hughes, Local refinement of analysis-suitable T-splines, Computer Methods in Applied Mechanics and Engineering 213-216 (2012) 206-222.

[9] L. Beirao da Veiga, A. Buffa, D. Cho, G. Sangalli, Analysis-suitable Tsplines are Dual-Compatible, Computer Methods in Applied Mechanics and Engineerinig 249-252 (2012) 42-51.

[10] D. R. Forsey, R. H. Bartels, Hierarchical B-spline refinement, Computer Graphics 22 (4) (1988) 205-212. 
[11] R. Kraft, Adaptive und linear unabhängige multilevel B-splines und ihre Anwendungen, PhD thesis, Universität Stuttgart, 1998.

[12] A.-V. Vuong, C. Giannelli, B. Jüttler, B. Simeon, A hierarchical approach to adaptive local refinement in isogeometric analysis, Computer Methods in Applied Mechanics and Engineering 200 (2011) 3554-3567.

[13] C. Giannelli, B. Jüttler, H. Speleers, THB-splines: The truncated basis for hierarchical splines, Computer Aided Geometric Design 29 (7) (2012) 485-498.

[14] T. Dokken, T. Lyche, K. F. Pettersen, Polynomial splines over locally refined box-partitions, Computer Aided Geometric Design 30 (3) (2013) 331-356.

[15] J. Deng, F. Chen, Y. Feng, Dimension of spline spaces over T-meshes, Journal of Computational and Applied Mathematics 194 (2006) 267-283.

[16] J. Deng, F. Chen, X. Li, C. Hu, W. Tong, Z. Yang, Y. Feng, Polynomial splines over hierarchical T-meshes, Graphical models 74 (2008) 76-86.

[17] X. Li, J. Deng, F. Chen, Surface modeling with polynomial splines over hierarchical T-meshes, Visual Computer 23 (2007) 1027-1033.

[18] N. Nguyen-Thanh, H. Nguyen-Xuan, S. Bordas, T. Rabczuk, Isogeometric analysis using polynomial splines over hierarchical T-meshes for two-dimensional elastic solids, Computer Methods in Applied Mechanics and Engineering 200 (2011) 1892-1908.

[19] L. L. Schumaker, L. Wang, Approximation power of polynomial splines on T-meshes, Computer Aided Geometric Design 29 (2012) 599-612.

[20] X. Li, F. Chen, On the instability in the dimension of splines spaces over T-meshes, Computer Aided Geometric Design 28 (2011) 420-426.

[21] D. Berdinsky, M. Oh, T. Kim, B. Mourrain, On the problem of instability in the dimension of a spline space over a T-mesh, Computers \& Graphics 36 (2012) 507-513.

[22] M. Wu, J. Deng, F. Chen, Dimension of spline spaces with highest order smoothness over hierarchical T-meshes, Computer Aided Geometric Design 30 (1) (2013) 20-34. 
[23] M. Wu, J. Xu, R. Wang, Z. Yang, Hierarchical bases of spline spaces with highest order smoothness over hierarchical T-subdivisions, Computer Aided Geometric Design 29 (7) (2012) 499-509.

[24] C. Giannelli, B. Jüttler, Bases and dimensions of bivariate hierarchical tensor-product splines, Journal of Computational and Applied Mathematics 239 (2013) 162-178.

[25] B. Mourrain, On the dimension of spline spaces on planar T-meshes, Mathematics of Computation, http://dx.doi.org/10.1090/S0025-57182013-02738-X.

[26] Z. Huang, J. Deng, Y. Feng, F. Chen, New proof of dimension formula of spline spaces over T-meshes via smoothing cofactors, Journal of Computational Mathematics 24 (4) (2006) 501-514.

[27] C.-J. Li, R.-H. Wang, F. Zhang, Improvement on the dimension of spline spaces on T-mesh, Journal of Information and Computational Science 3 (2) (2006) 235-244. 